\title{
Sampling Lissajous and Fourier Knots
}

\author{
Adam Boocher* \\ University of Notre Dame \\ Jay Daigle* \\ Pomona College \\ Jim Hoste* \\ Pitzer College \\ Wenjing Zheng* \\ University of California, Berkeley
}

July 25, 2018

\begin{abstract}
A Lissajous knot is one that can be parameterized as

$$
K(t)=\left(\cos \left(n_{x} t+\phi_{x}\right), \cos \left(n_{y} t+\phi_{y}\right), \cos \left(n_{z} t+\phi_{z}\right)\right)
$$
\end{abstract}

where the frequencies $n_{x}, n_{y}$, and $n_{z}$ are relatively prime integers and the phase shifts $\phi_{x}, \phi_{y}$ and $\phi_{z}$ are real numbers. Lissajous knots are highly symmetric, and for this reason, not all knots are Lissajous. We prove several theorems which allow us to place bounds on the number of Lissajous knot types with given frequencies and to efficiently sample all possible Lissajous knots with a given set of frequencies. In particular, we systematically tabulate all Lissajous knots with small frequencies and as a result substantially enlarge the tables of known Lissajous knots.

A Fourier- $(i, j, k)$ knot is similar to a Lissajous knot except that the $x, y$ and $z$ coordinates are now each described by a sum of $i, j$ and $k$ cosine functions respectively. According to Lamm, every knot is a Fourier- $(1,1, k)$ knot for some $k$. By randomly searching the set of Fourier- $(1,1,2)$ knots we find that all 2-bridge knots up to 14 crossings are either Lissajous or Fourier- $(1,1,2)$ knots. We show that all twist knots are Fourier- $(1,1,2)$ knots and give evidence suggesting that all torus knots are Fourier- $(1,1,2)$ knots.

As a result of our computer search, several knots with relatively small crossing numbers are identified as potential counterexamples to interesting conjectures.

\footnotetext{
*Supported by NSF REU grant DMS-0453284.
} 


\section{Introduction}

A Lissajous knot $K$ in $\mathbb{R}^{3}$ is a knot that has a parameterization $K(t)=(x(t), y(t), z(t))$ given by

$$
\begin{aligned}
& x(t)=\cos \left(n_{x} t+\phi_{x}\right) \\
& y(t)=\cos \left(n_{y} t+\phi_{y}\right) \\
& z(t)=\cos \left(n_{z} t+\phi_{z}\right)
\end{aligned}
$$

where $0 \leq t \leq 2 \pi, n_{x}, n_{y}$, and $n_{z}$ are integers, and $\phi_{x}, \phi_{y}, \phi_{z} \in \mathbb{R}$.

Lissajous knots were first studied in [1] where some of their elementary properties were established. Most notably, Lissajous knots enjoy a high degree of symmetry. In particular, if the three frequencies $n_{x}, n_{y}$ and $n_{z}$ (which must be pairwise relatively prime - see [1]) are all odd, then the knot is strongly plus amphicheiral. If one of the frequencies is even, then the knot is 2-periodic, with the additional property that it links its axis of rotation once. These symmetry properties imply (strictly) weaker properties such as the fact that the Alexander polynomial of a Lissajous knot must be a square mod 2, which in turn implies that its Arf invariant must be zero. See [1, 7] and [1] for details. Thus for example, the trefoil and figure eight knots are not Lissajous since their Arf invariants are one. In fact, "most" knots are not Lissajous.

To date it is unknown if every knot which is strongly plus amphicheiral or 2-periodic (and links its axis of rotation once) is Lissajous. Several knots with relatively few crossings exist which meet these symmetry requirements and yet are still unknown to be Lissajous or not. For example, according to [6] there are only three prime knots with 12 or less crossings which are strongly plus amphicheiral: 10a103 (1099), 10a121 $\left(10_{123}\right)$, and 12a427. Here we have given knot names in both the Dowker-Thistlethwaite ordering of the Hoste-Thistlethwaite-Weeks table [6] and, in parenthesis, the Rolfsen [14] ordering (for knots with 10 or less crossings). Symmetries of the knots in the Hoste-Thistlethwaite-Weeks table were computed using SnapPea as described in [6]. Of these three knots, only 10a103 $\left(10_{99}\right)$ was previously reported as Lissajous. (See [10] and [11.) However we find 12a427 to be Lissajous. (See Section 5 of this paper.) This leaves open the case of 10a121. As a further example, there are exactly four 8-crossings knots which are 2-bridge, 2-periodic, and link their axis of rotation once. Despite our extensive searching (see Section 5) only one of these knots turned up as Lissajous (and it had already been reported as such in [10). Whether the other three are Lissajous remains unknown.

Lissajous knots are a subset of the more general class of Fourier knots. A Fourier- $(i, j, k)$ knot is one that can be parameterized as

$$
\begin{aligned}
& x(t)=A_{x, 1} \cos \left(n_{x, 1} t+\phi_{x, 1}\right)+\ldots+A_{x, i} \cos \left(n_{x, i} t+\phi_{x, i}\right) \\
& y(t)=A_{y, 1} \cos \left(n_{y, 1} t+\phi_{y, 1}\right)+\ldots+A_{y, j} \cos \left(n_{y, j} t+\phi_{y, j}\right) \\
& z(t)=A_{z, 1} \cos \left(n_{z, 1} t+\phi_{z, 1}\right)+\ldots+A_{z, k} \cos \left(n_{z, k} t+\phi_{z, k}\right) .
\end{aligned}
$$

Because any function can be closely approximated by a sum of cosines, every knot is a Fourier knot for some $(i, j, k)$. But a remarkable theorem of Lamm [10] states that in fact every knot is a Fourier- $(1,1, k)$ knot for some $k$. While $k$ cannot equal one for all knots (these are the Lissajous knots, and not all knots are Lissajous) could $k$ possibly be less than some universal bound $M$ for all knots? This seems unlikely, with the more reasonable outcome being that $k$ depends on the specific knot $K$. Yet no one has found a knot for which $k$ must be bigger than two!

If $K$ is a Fourier- $(1,1, k)$ knot then its bridge number is less than or equal to the minimum of $n_{x}$ and $n_{y}$. (The bridge number of a knot $K$ can be defined as the smallest number of extrema on $K$ with respect to a given direction in $\mathbb{R}^{3}$, taken over all representations of $K$ and with respect to all directions. See [2] or 14] for more details.) Moreover, Lamm's proof is constructive and explicitly shows that if $K$ has bridge number $b$, then $K$ is a Fourier- $(1,1, k)$ knot for some $k$ and with $n_{x}=b$. This raises several interesting questions. For any knot $K$, when expressed as a Fourier- $(1,1, k)$ knot, can the minimum values of $n_{x}$ and $k$ 
be simultaneously realized? In particular, can a knot which is Lissajous and with bridge index $b$ be realized as a Lissajous knot with $n_{x}=b$ ?

Let $\mathcal{L}\left(n_{x}, n_{y}, n_{z}\right)$ be the set of all Lissajous knots with frequencies $n_{x}, n_{y}, n_{z}$. (Throughout this paper we consider a knot and its mirror image to be equivalent.) One of the main goals of this paper is to investigate the set $\mathcal{L}\left(n_{x}, n_{y}, n_{z}\right)$. By a simple change of variables, $t \rightarrow t+c$, we may alter the phase shifts. Therefore we will assume that $\phi_{x}=0$ in all that follows. This leaves the pair of parameters $\left(\phi_{y}, \phi_{z}\right)$ which vary within the phase torus $[0,2 \pi] \times[0,2 \pi]$. In Section 2 we examine the phase torus and identify a finite number of regions in which the phase shifts must lie, with each region corresponding to a single knot type. We further show that a periodic pattern of knot types are produced as one traverses the phase torus. This allows us to prove

Theorem 1. Let $\left|\mathcal{L}\left(n_{x}, n_{y}, n_{z}\right)\right|$ be the number of distinct Lissajous knots with frequencies $\left(n_{x}, n_{y}, n_{z}\right)$. Then

$$
\left|\mathcal{L}\left(n_{x}, n_{y}, n_{z}\right)\right| \leq 2 n_{x} n_{y} .
$$

If furthermore $n_{x}=2$, then

$$
\left|\mathcal{L}\left(2, n_{y}, n_{z}\right)\right| \leq 2 n_{y}+1
$$

There is also a periodicity that exists across frequencies and in Section 2 we also prove

Theorem 2. $\mathcal{L}\left(n_{x}, n_{y}, n_{z}\right) \subseteq \mathcal{L}\left(n_{x}, n_{y}, n_{z}+2 n_{x} n_{y}\right)$, with equality if $n_{z} \geq 2 n_{x} n_{y}-n_{y}$.

Our analysis of the phase torus, together with these theorems allow us to efficiently sample (with the aid of a computer) all possible Lissajous knots having two of the three frequencies bounded. Even with relatively small frequencies, the three natural projections of a Lissajous knot into the three coordinate planes can have a large number of crossings. (The projection into the $x y$-plane has $2 n_{x} n_{y}-n_{x}-n_{y}$ crossings.) With frequencies of 10 or more, diagrams with hundreds of crossings result and many, if not most, knot invariants are computationally out of reach. Thus it becomes extremely difficult to compare different Lissajous knots with large frequencies, or to try to locate them in existing knot tables. However, if one frequency is two, the knot is 2-bridge and even with hundreds of crossings it is relatively simple to compute the identifying fraction $p / q$ by which 2-bridge knots are classified.

In Section 2 we recall basic facts about Lissajous knots and prove several theorems, including the two already mentioned, that will allow us to efficiently sample all Lissajous knots with two given frequencies. In Section 4 we then recall some basic facts about 2-bridge knots. Using these results we then report in Section 5 on our computer experiments. Theorems similar to those given in Section 2 but for Fourier- $(1,1, k)$ knots would necessarily be much more complicated and we only begin the analysis of the phase torus for Fourier- $(1,1,2)$ knots in Section 3. Without the analogous results, we have not been able to rigorously sample Fourier knots. Instead, we have proceeded by two methods, either random sampling, or a sampling based on first forming a bitmap image of the phase torus and its singular curves. However, even without exhaustive sampling, our data show that all 2-bridge knots up to 14 crossings are Fourier- $(1,1, k)$ knots with $k \leq 2$ and with $n_{x}=2$.

This research was carried out at the Claremont College's REU program in the summer of 2006. The authors thank the National Science Foundation and the Claremont Colleges for their generous support.

\section{The Phase Torus-Lissajous Knots}

Suppose $K(t)$ is a Lissajous knot and consider its diagram in the $x y$-plane. Each crossing in this diagram corresponds to a double point in the $x y$-projection given by a pair of times $\left(t_{1}, t_{2}\right)$, where $x\left(t_{1}\right)=x\left(t_{2}\right)$ and $y\left(t_{1}\right)=y\left(t_{2}\right)$. The following lemma is given in [7. 


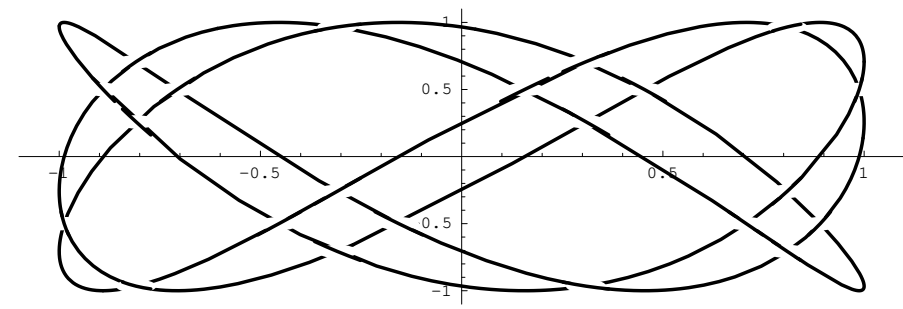

Figure 1: A Lissajous knot with frequencies $(3,5,7)$ and corresponding phase shifts $(0, \pi / 4, \pi / 12)$. The Type I crossings appear in two rows with five crossings each and the Type II crossings appear in four columns with three crossings each.

Lemma 3. Let $K(t)$ be a Lissajous knot. There are two types of time pairs $\left(t_{1}, t_{2}\right)$ that give double points in the $x y$-projection:

$$
\begin{array}{rr}
\text { Type } I: \quad\left(t_{1}, t_{2}\right)=\quad\left(\left(-\frac{k}{n_{x}}+\frac{j}{n_{y}}\right) \pi-\frac{\phi_{y}}{n_{y}},\left(\frac{k}{n_{x}}+\frac{j}{n_{y}}\right) \pi-\frac{\phi_{y}}{n_{y}}\right) \\
1 \leq k \leq n_{x}-1, \quad 1+\left\lfloor\frac{n_{y}}{n_{x}} k+\frac{\phi_{y}}{\pi}\right\rfloor \leq j \leq\left\lfloor 2 n_{y}-\frac{n_{y}}{n_{x}} k+\frac{\phi_{y}}{\pi}\right\rfloor \\
\text { Type II: } \quad\left(t_{1}, t_{2}\right)=\quad\left(\left(-\frac{k}{n_{y}}+\frac{j}{n_{x}}\right) \pi-\frac{\phi_{x}}{n_{x}},\left(\frac{k}{n_{y}}+\frac{j}{n_{x}}\right) \pi-\frac{\phi_{x}}{n_{x}}\right) \\
1 \leq k \leq n_{y}-1, \quad 1+\left\lfloor\frac{n_{x}}{n_{y}} k+\frac{\phi_{x}}{\pi}\right\rfloor \leq j \leq\left\lfloor 2 n_{x}-\frac{n_{x}}{n_{y}} k+\frac{\phi_{x}}{\pi}\right\rfloor
\end{array}
$$

There are $n_{x} n_{y}-n_{y}$ double points of Type I, and $n_{x} n_{y}-n_{x}$ double points of Type II.

Figure 1 1 shows a Lissajous knot with frequencies $(3,5,7)$ and corresponding phase shifts $(0, \pi / 4, \pi / 12)$. Since all the frequencies are odd, this knot is symmetric through the origin. It is not hard to show that in general, the Type I crossings line up in sets of size $n_{y}$ on $n_{x}-1$ horizontal lines, while the Type II crossings line up in sets of size $n_{x}$ on $n_{y}-1$ vertical lines. If $n_{x}=2$ there is a single row of Type I crossings, all of which lie on the $x$-axis and $n_{y}-1$ columns of Type II crossings with each column consisting of two crossings.

Not all phase shift pairs will generate curves that are knots. Assuming $\phi_{x}=0$, the knot $K(t)$ will intersect itself, and thus fail to be a knot, exactly when the phase shifts satisfy

$$
\begin{aligned}
\phi_{z} & =\frac{n_{z}}{n_{y}} \phi_{y}+l \frac{\pi}{n_{y}}, \text { or } \\
\phi_{z} & =l \frac{\pi}{n_{x}}, \text { or } \\
\phi_{y} & =l \frac{\pi}{n_{x}}
\end{aligned}
$$

for some integer $l$. Crossings of Type I become singular precisely when Equation 1 holds; crossings of Type II when Equaton 2 holds. When Equation 3 holds, the entire $x y$-projection degenerates to an arc. While this alone does not imply the knot has points of self-intersection, this is indeed the case. See [8, 1, 7, for more details. In Proposition 5, we specifically identify which crossings become singular as the phase shifts move across these lines.

The slanted, horizontal and vertical lines given in Equations 13 obviously divide the phase torus into regions with each region defining one knot type. Thus there are only a finite number of knots types possible for a given set of frequencies. There is, however, a great deal of repetition in knot types as one traverses the phase torus due to the periodicity of the cosine function. The following theorem describes a nice choice of "fundamental domain" on the phase torus to which we may restrict our attention. 
Theorem 4. Any knot in $\mathcal{L}\left(n_{x}, n_{y}, n_{z}\right)$ can be represented with $\phi_{x}=0$ and using some phase shift pair $\left(\phi_{y}, \phi_{z}\right)$ in $\left[0, \frac{\pi}{n_{x}}\right] \times[0, \pi]$.

Proof. Define an equivalence relation $\sim$ on the phase torus for $\mathcal{L}\left(n_{x}, n_{y}, n_{z}\right)$ by $\left(\phi_{y}, \phi_{z}\right) \sim\left(\phi_{y}^{\prime}, \phi_{z}^{\prime}\right)$ if the Lissajous knot with phase shifts $\left(0, \phi_{y}, \phi_{z}\right)$ is the same as the knot with phase shifts $\left(0, \phi_{y}^{\prime}, \phi_{z}^{\prime}\right)$, or its mirror image. Clearly

$$
\left(\phi_{y}, \phi_{z}\right) \sim\left(\phi_{y} \pm \pi, \phi_{z}\right) \sim\left(\phi_{y}, \phi_{z} \pm \pi\right) .
$$

If $K \in \mathcal{L}\left(n_{x}, n_{y}, n_{z}\right)$, a change of variable $t \rightarrow t+\frac{\pi}{n_{x}}$ shows that $K$ is also parameterized as

$$
\begin{aligned}
& x=-\cos \left(n_{x} t\right) \\
& y=\cos \left(n_{y} t+\phi_{y}+\frac{n_{y} \pi}{n_{x}}\right) \\
& z=\cos \left(n_{z} t+\phi_{z}+\frac{n_{z} \pi}{n_{x}}\right) .
\end{aligned}
$$

Therefore we also have

$$
\left(\phi_{y}, \phi_{z}\right) \sim\left(\phi_{y}+\frac{n_{y} \pi}{n_{x}}, \phi_{z}+\frac{n_{z} \pi}{n_{x}}\right) .
$$

Now since $n_{x}$ and $n_{y}$ are relatively prime, there are integers $k$ and $l$ with $0 \leq \phi_{y}+\frac{k n_{y} \pi}{n_{x}}-l \pi<\frac{\pi}{n_{x}}$. Repeatedly using (4) and (5) we obtain

$$
\left(\phi_{y}, \phi_{z}\right) \sim\left(\phi_{y}+\frac{k n_{y} \pi}{n_{x}}-l \pi, \phi_{z}+\frac{k n_{z} \pi}{n_{x}}\right)
$$

The first coordinate is already in $\left[0, \frac{\pi}{n_{x}}\right]$; we can shift the second coordinate by multiples of $\pi$ until it is in $[0, \pi]$. Thus an arbitrary point $\left(\phi_{y}, \phi_{z}\right)$ is equivalent to some point in $\left[0, \frac{\pi}{n_{x}}\right] \times[0, \pi]$, as desired.

Figure 2 shows the fundamental domain on the phase torus for $\mathcal{L}(2,3,5)$. The singular lines divide the domain into regions with each region determining a single knot type. Since these knots are all 2-bridge, we identify each with its classifying fraction $p / q$.

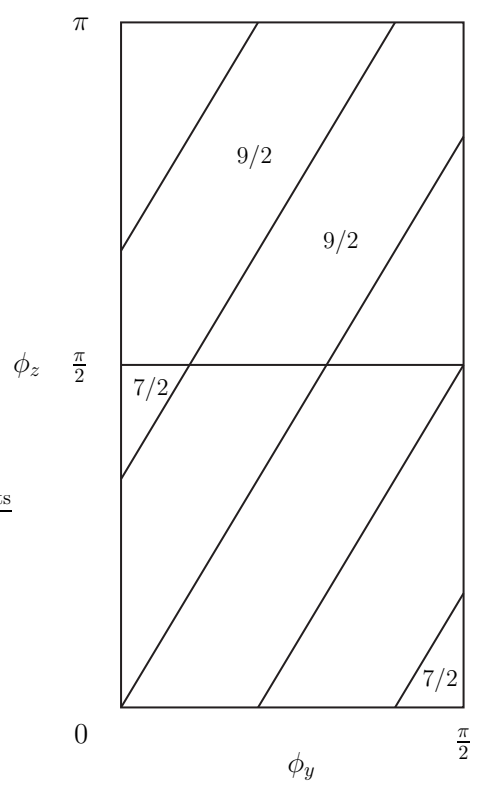

Figure 2: The fundamental domain of the phase torus for $\mathcal{L}(2,3,5)$. Each region defines a single 2-bridge knot which is identified by its classifying fraction $p / q$. Unlabeled regions define unknots. 
Our next result specifically describes what happens as we cross a singular line of the type given in Equation 1 or 2 .

Proposition 5. Let $K$ and $K^{\prime}$ be two Lissajous knots with frequencies $\left(n_{x}, n_{y}, n_{z}\right)$ and phase shifts ( $\left.\phi_{y}, \phi_{z}\right)$ and $\left(\phi_{y}^{\prime}, \phi_{z}^{\prime}\right)$ respectively.

1. Suppose $\left(\phi_{y}, \phi_{z}\right)$ and $\left(\phi_{y}^{\prime}, \phi_{z}^{\prime}\right)$ lie in two adjacent regions of the phase torus separated by a diagonal line $L$ given by $\phi_{z}=\frac{n_{z}}{n_{y}} \phi_{y}+l \frac{\pi}{n_{y}}$. Then $K$ and $K^{\prime}$ differ by changing all Type $I$ crossings with parameters $(k, j)$ such that with $j n_{z}+l \equiv 0 \bmod n_{y}$. The number of such crossings is $n_{x}-1$.

2. Suppose $\left(\phi_{y}, \phi_{z}\right)$ and $\left(\phi_{y}^{\prime}, \phi_{z}^{\prime}\right)$ lie in two adjacent regions of the phase torus separated by a horizontal line $L$ given by $\phi_{z}=l \frac{\pi}{n_{x}}$. Then $K$ and $K^{\prime}$ differ by changing all Type II crossings with parameters $(k, j)$ such that $j n_{z}+l \equiv 0 \bmod n_{x}$. The number of such crossings is $n_{y}-1$.

Proof. If $\left(t_{1}, t_{2}\right)$ is a Type I crossing with parameters $(k, j)$, then $z\left(t_{1}\right)=z\left(t_{2}\right)$ if and only if

$$
\cos \left(n_{z} t_{1}+\phi_{z}\right)=\cos \left(n_{z} t_{2}+\phi_{z}\right)
$$

which will occur if and only if

$$
n_{z}\left(t_{1}-t_{2}\right)=2 m \pi \quad \text { or } \quad n_{z}\left(t_{1}+t_{2}\right)+2 \phi_{z}=2 m^{\prime} \pi
$$

for some integers $m, m^{\prime}$. For Type I crossings,

$$
t_{1}-t_{2}=-\frac{2 k}{n_{x}} \pi \quad \text { and } \quad t_{1}+t_{2}=\frac{2 j}{n_{y}} \pi-\frac{2 \phi_{y}}{n_{y}} .
$$

If (6) is to hold, then in the first case, we have

$$
-\frac{2 k n_{z}}{n_{x}} \pi=2 m \pi
$$

which is equivalent to $-k n_{z}=m n_{x}$. This is impossible since $n_{x}$ and $n_{z}$ are relatively prime and $1 \leq k \leq$ $n_{x}-1$.

In the second case, we have

$$
n_{z}\left(2 \frac{j}{n_{y}} \pi-2 \frac{\phi_{y}}{n_{y}}\right)+2 \phi_{z}=2 m^{\prime} \pi
$$

which is equivalent to

$$
\phi_{z}=\frac{n_{z}}{n_{y}} \phi_{y}+\left(m^{\prime} n_{y}-j n_{z}\right) \frac{\pi}{n_{y}} .
$$

Thus Type I crossings only become singular on lines of the form given in Equation 1 with $l=m n_{y}-j n_{z}$.

If $\phi_{z}=\frac{n_{z}}{n_{x}} \phi_{y}+l \frac{\pi}{n_{y}}+\varepsilon$ and $j n_{z}+l=m n_{y}$ for some integer $m$ then it is straightforward to check that

$$
z\left(t_{1}\right)-z\left(t_{2}\right)=(-1)^{m} 2 \sin \varepsilon \sin \frac{k n_{z} \pi}{n_{x}} .
$$

Hence, as we move across the line $L$ by letting $\varepsilon$ go from a small positive value to a small negative value, the difference $z\left(t_{1}\right)-z\left(t_{2}\right)$ changes sign. Thus the Type I crossings with parameters $(k, j)$ actually change from over to under or vice versa, rather than simply becoming singular and then "rebounding" to their original positions.

Finally, note that once $l$ is fixed this does not necessarily uniquely determine $j$ and thus the corresponding Type I crossing. If both $j n_{z}+l \equiv 0 \bmod n_{y}$ and $j^{\prime} n_{z}+l \equiv 0 \bmod n_{y}$ then $j \equiv j^{\prime} \bmod n_{y}$ since $n_{y}$ and $n_{z}$ are 
relatively prime. If $n_{x}=2$, then $k=1$ and $j$ lies in an interval of length $n_{y}$. Thus with $n_{x}=2$ we have that $j$ is uniquely determined by $l$ and a single crossing changes as we move across $L$. But if $n_{x}>2$ and $k=1$ then $j$ lies in an interval of length greater than $n_{y}$. Hence two admissible values, $j$ and $j+n_{y}$, are possible. Using $j$, we must have $1 \leq k \leq\left\lfloor\frac{n_{x}}{n_{y}}\left(j-\frac{\phi_{y}}{\pi}\right)\right\rfloor$ while for $j+n_{y}$ we must have $1 \leq k \leq\left\lfloor\frac{n_{x}}{n_{y}}\left(-j+n_{y}+\frac{\phi_{y}}{\pi}\right)\right\rfloor$. Thus the total number of possible points $(k, j)$ is $\left\lfloor\frac{n_{x}}{n_{y}}\left(j-\frac{\phi_{y}}{\pi}\right)\right\rfloor+\left\lfloor\frac{n_{x}}{n_{y}}\left(-j+n_{y}+\frac{\phi_{y}}{\pi}\right)\right\rfloor=n_{x}-1$.

A similar discussion handles the Type II crossings.

Corollary 6. Suppose $K$ and $K^{\prime}$ are Lissajous knots with frequencies $\left(n_{x}, n_{y}, n_{z}\right)$ and phase shifts which belong to regions separated by $2 n_{y}$ singular lines of the type given in Equation 1. Then all Type I crossings are the same for both knots.

Proof. From Proposition [ 5 we know that crossing the line $\phi_{z}=\frac{n_{z}}{n_{y}} \phi_{y}+l \frac{\pi}{n_{y}}$ changes exactly those Type I crossings with parameters $(k, j)$ for which $j n_{z}+l \equiv 0 \bmod n_{y}$. Thus if we cross the singular line corresponding to $l$ and then later cross the line corresponding to $l+n_{y}$ the same set of Type I crossings will first be changed and then changed back again. Hence, after crossing over $2 n_{y}$ such lines all Type I crossings will be restored to their original position.

If $n_{x}=2$ there is even more repetition due to additional symmetry as is shown in the following result.

Proposition 7. Let $K$ and $K^{\prime}$ be Lissajous knots with frequencies $\left(2, n_{y}, n_{z}\right)$ and phase shifts $\left(\phi_{y}, \phi_{z}\right)$ and $\left(\phi_{y}^{\prime}, \phi_{z}^{\prime}\right)$ respectively. If $\left(\phi_{y}, \phi_{z}\right)$ and $\left(\phi_{y}^{\prime}, \phi_{z}^{\prime}\right)$ are symmetric with respect to the point $(\pi / 4, \pi / 4)$ or the point $(\pi / 4,3 \pi / 4)$ then $K$ and $K^{\prime}$ are equivalent.

Proof. Suppose $\left(\phi_{y}, \phi_{z}\right)$ and $\left(\phi_{y}^{\prime}, \phi_{z}^{\prime}\right)$ are symmetric with respect to the point $(\pi / 4, \pi / 4)$. Then $\phi_{y}^{\prime}=\pi / 2-\phi_{y}$ and $\phi_{z}^{\prime}=\pi / 2-\phi_{z}$. Thus

$$
\begin{aligned}
K^{\prime}(-t+\pi / 2) & =\left(\cos (-2 t+\pi), \cos \left(-n_{y} t+n_{y} \pi / 2+\pi / 2-\phi_{y}\right), \cos \left(-n_{z} t+n_{z} \pi / 2+\pi / 2-\phi_{z}\right)\right. \\
& =\left(-\cos (2 t),(-1)^{\left(n_{y}+1\right) / 2} \cos \left(n_{y} t+\phi_{y}\right),(-1)^{\left(n_{z}+1\right) / 2} \cos \left(n_{z} t+\phi_{z}\right)\right)
\end{aligned}
$$

which is either $K(t)$ or its mirror image $\bar{K}(t)$.

The second case follows similarly.

We may now prove Theorem 1

Proof of Theorem 1: The fundamental domain is divided into $n_{x}$ "boxes" of the form $\left[0, \frac{\pi}{n_{x}}\right] \times\left[k \frac{\pi}{n_{x}},(k+1) \frac{\pi}{n_{x}}\right]$ for $0 \leq k \leq n_{x}-1$. Within each box all the knots have the same Type II crossings and hence by Corollary 6 there are at most $2 n_{y}$ different knot types in that box. Since there are $n_{x}$ boxes we obtain at most $2 n_{x} n_{y}$ different knots.

If $n_{x}=2$ there is the additional rotational symmetry in each box given by Proposition 7 The center of each box either lies on a slanted singular line, or midway between two such lines. Moreover, one of the two boxes will be one way and the other box will be the other way. There are at most $n_{y}$ knot types in the box where the center of the box lies on a singular line, and there are at most $n_{y}+1$ knot types in the box otherwise. Thus there are at most $2 n_{y}+1$ knot types in total. 
Our results thus far allow us to efficiently sample all Lissajous knots with a given set of frequencies $\left(n_{x}, n_{y}, n_{z}\right)$. We can easily pick one set of phase shifts from each region on the phase torus and Corollary 6] and Proposition 7 in the case when $n_{x}=2$, allows us to further restrict the regions that we must sample. However, once $n_{x}, n_{y}$, and $\phi_{y}$ are given, the $x y$-projection has been fixed and it is natural to ask if all possible choices for $n_{z}$ are necessary. Theorem 2, which is stated in the introduction, shows that in fact, only a finite number of values for $n_{z}$ are needed to produce all possible knots.

Proof of Theorem 2; Suppose that $K \in \mathcal{L}\left(n_{x}, n_{y}, n_{z}\right), K^{\prime} \in \mathcal{L}\left(n_{x}, n_{y}, n_{z}+2 n_{x} n_{y}\right)$ and that both knots have the same phase shifts. We will show first that each knot has its Type II crossings arranged the same way.

Let $\left(t_{1}, t_{2}\right)$ be a Type II crossing with parameters $(k, j)$ and let

$$
\begin{aligned}
\Delta_{I I}\left(n_{x}, n_{y}, n_{z}, \phi_{z}, k, j\right) & =\cos \left(n_{z} t_{1}+\phi_{z}\right)-\cos \left(n_{z} t_{1}+\phi_{z}\right) \\
& =2 \sin \left(n_{z}\left(\frac{t_{1}+t_{2}}{2}\right)+\phi_{z}\right) \sin \left(n_{z}\left(\frac{t_{1}-t_{2}}{2}\right)\right) \\
& =-2 \sin \left(n_{z} \frac{j \pi}{n_{x}}+\phi_{z}\right) \sin \left(n_{z} \frac{k \pi}{n_{y}}\right)
\end{aligned}
$$

be the height difference between the two points on the knot directly above the crossing.

It is easy to verify that

$$
\Delta_{I I}\left(n_{x}, n_{y}, n_{z}, \phi_{z}, k, j\right)=\Delta_{I I}\left(n_{z}+2 n_{x} n_{y}, n_{y}, n_{z}, \phi_{z}, k, j\right) \text { for all } k, j .
$$

Thus if $n_{z}$ is increased by $2 n_{x} n_{y}$, not only do all Type II crossings remain unchanged, they each maintain the same height difference between upper and lower strand.

We now shift our focus to Type I crossings. Let $K$ have phase shifts $\left(\phi_{y}, \frac{n_{z}}{n_{y}} \phi_{y}-\varepsilon\right)$ and choose $\varepsilon$ small enough so that $K$ corresponds to the region just below the singular line $\phi_{z}=\frac{n_{z}}{n_{y}} \phi_{y}$. Let $K^{\prime}$ correspond to the "same" region, that is, let $K^{\prime}$ have phase shifts $\left(\phi_{y}, \frac{n_{z}+2 n_{x} n_{y}}{n_{y}} \phi_{y}-\varepsilon\right)$. As before, let $\left(t_{1}, t_{2}\right)$ be a Type I crossing with parameters $(k, j)$ and let

$$
\begin{aligned}
\Delta_{I}\left(n_{x}, n_{y}, n_{z}, \phi_{y}, \phi_{z}, k, j\right) & =\cos \left(n_{z} t_{1}+\phi_{z}\right)-\cos \left(n_{z} t_{1}+\phi_{z}\right) \\
& =2 \sin \left(n_{z}\left(\frac{t_{1}+t_{2}}{2}\right)+\phi_{z}\right) \sin \left(n_{z}\left(\frac{t_{1}-t_{2}}{2}\right)\right) \\
& =-2 \sin \left(n_{z} \frac{j \pi}{n_{y}}-\frac{n_{z} \phi_{y}}{n_{y}}+\phi_{z}\right) \sin \left(n_{z} \frac{k \pi}{n_{x}}\right)
\end{aligned}
$$

be the height difference between the two points on the knot directly above the crossing. It is easy to check that

$$
\Delta\left(n_{x}, n_{y}, n_{z}, \frac{n_{z}}{n_{y}} \phi_{y}-\varepsilon, k, j\right)=\Delta\left(n_{x}, n_{y}, n_{z}+2 n_{x} n_{y}, \frac{n_{z}+2 n_{x} n_{y}}{n_{y}} \phi_{y}-\varepsilon, k, j\right) .
$$

Thus $K$ and $K^{\prime}$ are the same knot since both the Type I and Type II crossings are arranged the same way in each. If the phase shifts for $K$ are now changed by moving into an adjacent region, and if the phase shifts for $K^{\prime}$ are changed in the same way, then the same set of crossings is changed for both $K$ and $K^{\prime}$ and hence $K$ and $K^{\prime}$ remain the same knot. Therefore

$$
\mathcal{L}\left(n_{x}, n_{y}, n_{z}\right) \subseteq \mathcal{L}\left(n_{x}, n_{y}, n_{z}+2 n_{x} n_{y}\right)
$$

According to Corollary [6, the pattern of knot types in each square $\left[0, \pi / n_{x}\right] \times\left[k \pi / n_{x},(k+1) \pi / n_{x}\right]$, as we move from the upper left corner to the lower right corner, is periodic with period $2 n_{y}$. Thus if each box contains 
at least $2 n_{y}$ regions the inclusion in (7) is equality. Now the distance between successive singular lines of the type given in Equation 1 is $\frac{\pi}{\sqrt{n_{y}^{2}+n_{z}^{2}}}$ and the distance between lines of slope $\frac{n_{z}}{n_{y}}$ containing opposite corners of the square is $\frac{\left(n_{2}+n_{3}\right) \pi}{n_{1} \sqrt{n_{y}^{2}+n_{z}^{2}}}$. Thus there are at least $\left\lfloor\frac{n_{y}+n_{z}}{n_{x}}\right\rfloor$ regions in each square. Hence the inclusion in (77) is equality if $2 n_{y} \leq\left\lfloor\frac{n_{y}+n_{z}}{n_{x}}\right\rfloor$. It is easy to check that this is true if $n_{z} \geq 2 n_{x} n_{y}-n_{y}$.

Definition 1. For relatively prime integers $n_{x}$ and $n_{y}$ let $\mathcal{L}\left(n_{x}, n_{y}\right)=\bigcup_{n_{z} \in \mathbb{N}} \mathcal{L}\left(n_{x}, n_{y}, n_{z}\right)$.

Theorem 8. Let $n_{x}, n_{y}$ be relatively prime integers. Then

$$
\left|\mathcal{L}\left(n_{x}, n_{y}\right)\right| \leq 4 n_{x} n_{y}\left(n_{x}-1\right)\left(n_{y}-1\right)
$$

If furthermore $n_{x}=2$, then

$$
\left|\mathcal{L}\left(2, n_{y}\right)\right| \leq 2\left(n_{y}-1\right)\left(2 n_{y}+1\right)
$$

Proof. For fixed $n_{x}, n_{y}$ we need only consider $2 n_{x} n_{y}$ consecutive values of $n_{z}$. To count the number of values that are relatively prime to both $n_{x}$ and $n_{y}$ we first subtract $2 n_{y}$ multiples of $n_{x}$ that lie in that range as well as $2 n_{x}$ multiples of $n_{y}$ and then add back in the 2 multiples of $n_{x} n_{y}$. Thus the number of possible values of $n_{z}$ is bounded above by $2 n_{x} n_{y}-2 n_{x}-2 n_{y}+2=2\left(n_{x}-1\right)\left(n_{y}-1\right)$ and applying Theorem 1 yields the result.

\section{The Phase Torus-Fourier- $(1,1,2)$ Knots}

The phase torus of a Fourier- $(1,1, k)$ is, in general, $k+2$ dimensional although we may set any one phase shift equal to zero and drop to a $k+1$ dimension space. If $k=2, \phi_{x}=0$ and we fix $\phi_{y}$, then we may again think of the 2-dimensional phase torus associated to the pair $\left(\phi_{z, 1}, \phi_{z, 2}\right)$. The singular curves are now much more complicated than in the Lissajous case, but can still be carefully described.

Suppose $K$ is a Fourier- $(1,1,2)$ knot with parameterization

$$
\begin{aligned}
& x(t)=\cos \left(n_{x} t\right) \\
& y(t)=\cos \left(n_{y} t+\phi_{y}\right) \\
& z(t)=\cos \left(n_{z, 1} t+\phi_{z, 1}\right)+A_{z, 2} \cos \left(n_{z, 2} t+\phi_{z, 2}\right) .
\end{aligned}
$$

Note that by rescaling we may assume that three of the four amplitudes are 1.

In the Lissajous case, we require that the three frequencies be pairwise relatively prime. The same proof (see [1]) can be used now to conclude that the three integers $n_{x}, n_{y}$ and $\operatorname{gcd}\left(n_{z, 1}, n_{z, 2}\right)$ must be pairwise relatively prime. This rules out several of the 16 cases that arise by considering all possible parities for the frequencies. Some of the remaining cases still give rise to highly symmetric knots, such as when all the frequencies are odd. In this case the knot is strongly plus amphicheiral just as in the Lissajous setting. But some of the parity cases produce knots with no apparent symmetry, suggesting that the set of Fourier- $(1,1,2)$ is much richer than the set of Lissajous knots.

We will not undertake an exhaustive analysis of the phase torus of Fourier- $(1,1,2)$ knots. Instead we offer a glimpse of the situation in the following Proposition which could be stated much more precisely. In particular, the constants in the statement of the proposition all depend on the pair of indices $(k, j)$ associated to either a Type I or II crossing. The interested reader can easily determine the constants by going through the details of the proof. Results analogous to Propositions 5 and 7 seem much harder. 
Proposition 9. Let $K$ be a Fourier-(1,1,2) knot with parameterization as given in 8 . Then the singular curves on the phase torus are of four possible types:
1. Lines of the form $\phi_{z, 2}=c$,
2. Lines of the form $\phi_{z, 1}=c$,
3. Lines of the form $\phi_{z, 2}= \pm \phi_{z, 1}+c$,
4. Curves with the shape of $\sin \left(\phi_{z, 2}\right)=c \sin \left(\phi_{z, 1}\right)$

where $c$ is a constant that, in the last case, is neither 0 nor \pm 1 .

Proof. Suppose that $\left(t_{1}, t_{2}\right)$ are a pair of times that produce a double point in the $x y$-projection of $K$. Using the identity $\cos x-\cos y=-2 \sin \left(\frac{x+y}{2}\right) \sin \left(\frac{x-y}{2}\right)$ we obtain

$$
z\left(t_{1}\right)-z\left(t_{2}\right)=-2 \sin \left(n_{z, 1} \frac{t_{1}+t_{2}}{2}+\phi_{z, 1}\right) \sin \left(n_{z, 1} \frac{t_{1}-t_{2}}{2}\right)-2 A \sin \left(n_{z, 2} \frac{t_{1}+t_{2}}{2}+\phi_{z, 2}\right) \sin \left(n_{z, 2} \frac{t_{1}-t_{2}}{2}\right) .
$$

We are interested in those values of $\phi_{z, 1}$ and $\phi_{z, 2}$ that make this difference zero.

Suppose now that $\left(t_{1}, t_{2}\right)$ define a Type II crossing with indices $(k, j)$. Then

$$
\begin{aligned}
& \frac{t_{1}+t_{2}}{2}=\frac{j \pi}{n_{x}} \\
& \frac{t_{1}-t_{2}}{2}=-\frac{k \pi}{n_{y}}
\end{aligned}
$$

and the crossing is singular if

$$
\sin \left(\frac{n_{z, 1} j \pi}{n_{x}}+\phi_{z, 1}\right) \sin \left(\frac{n_{z, 1} k \pi}{n_{y}}\right)=-A \sin \left(\frac{n_{z, 2} j \pi}{n_{x}}+\phi_{z, 2}\right) \sin \left(\frac{n_{z, 2} k \pi}{n_{y}}\right) .
$$

We are now led to several cases.

Case I: $n_{y} \mid n_{z, 1} k$

If $n_{y}$ divides $n_{z, 1}$ then we must have that $\sin \left(\frac{n_{z, 2 j \pi}}{n_{x}}+\phi_{z, 2}\right)=0$ since $k<n_{y}$ and $n_{y}, n_{z, 1}$ and $n_{z, 2}$ cannot have a common factor. This means that

$$
\phi_{z, 2}=m \pi-\frac{n_{z, 2} j \pi}{n_{x}}
$$

for some integer $m$.

Case II: $n_{y} \mid n_{z, 2} k$

This is similar to Case I leading to

$$
\phi_{z, 1}=m \pi-\frac{n_{z, 1} j \pi}{n_{x}}
$$

for some integer $m$.

If the first two cases do not occur, then we may rewrite Equation 9 as

$$
\sin \left(\frac{n_{z, 1} j \pi}{n_{x}}+\phi_{z, 1}\right)=C \sin \left(\frac{n_{z, 2} j \pi}{n_{x}}+\phi_{z, 2}\right)
$$




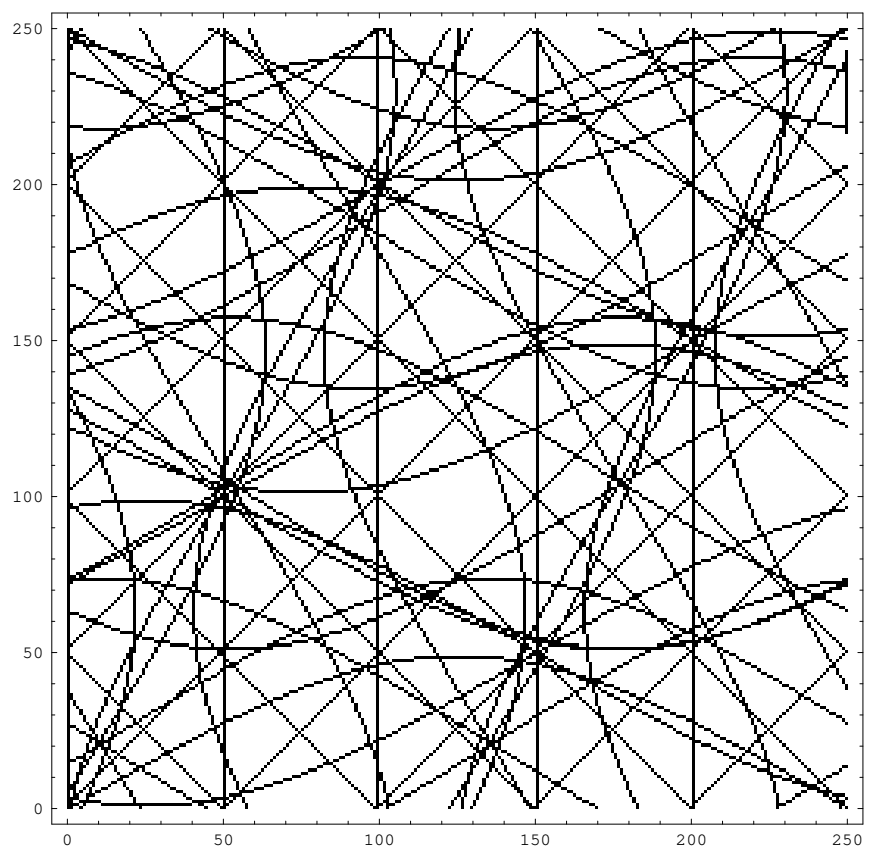

Figure 3: The phase torus for the Fourier- $(1,1,2)$ knot with $n_{x}=5, n_{y}=6, n_{z, 1}=1, n_{z, 2}=2, \phi_{x}=0, \phi_{y}=$ $\pi / 4$ and $A_{z, 1}=1$, shown for $0 \leq \phi_{z, 1} \leq \pi$ and $0 \leq \phi_{z, 2} \leq \pi$.

where

$$
C=-A \sin \left(\frac{n_{z, 2} k \pi}{n_{y}}\right) / \sin \left(\frac{n_{z, 1} k \pi}{n_{y}}\right)
$$

Case III: $|C|=1$

In this case we must have

$$
\left(\frac{n_{z, 1} j \pi}{n_{x}}+\phi_{z, 1}\right) \pm\left(\frac{n_{z, 2} j \pi}{n_{x}}+\phi_{z, 2}\right)=m \pi
$$

for some integer $m$, where the parity of $m$ depends on the sign of $C$ and whether we are forming a sum or difference in Equation 10, Thus $\phi_{z, 2}= \pm \phi_{z, 1}+c$ for some constant $c$.

Case IV: $|C| \neq 1$

In this case we are left with a translate of the curve

$$
\sin \left(\phi_{z, 1}\right)=C \sin \left(\phi_{z, 2}\right) .
$$

This is an interesting curve which, at first glance, appears much like a sine curve. It is oriented either vertically or horizontally depending on the value of $|C|$.

The analysis of a Type I crossing is similar and is left to the reader.

In Figure 3 we give an example showing a 250 pixel by 250 pixel bitmap image of the phase torus for a specific set of parameters. Even with relatively small frequencies, one can begin to appreciate the difficulty of systematically sampling each region of the phase torus for an arbitrary Fourier- $(1,1,2)$ knot. 


\section{2-Bridge Knots}

Every 2-bridge knot can be classified by a pair of relatively prime integers $(p, q)$ such that $p$ is odd and $0<q<p$. We will often write the pair $(p, q)$ as the fraction $p / q$. If $K_{p / q}$ and $K_{p^{\prime} / q^{\prime}}$ are two 2-bridge knots with corresponding fractions $p / q$ and $p^{\prime} / q^{\prime}$ then they are equivalent knots if and only if $p=p^{\prime}$ and $\pm q^{\prime} q^{ \pm 1} \equiv 1 \bmod p$. The reader is referred to [2] for details.

If $K$ is a Fourier $(1,1,2)$ knot with $n_{x}=2$ then $K$ is a 2-bridge knot. We may recover the fraction $p / q$ from the Lissajous projection in the $x y$-plane as follows. This projection is a 4-plat diagram. As we move in the $x$-direction from left to right we see a single Type I crossings on the $x$-axis, then a pair of Type II crossings which are symmetric with respect to the $x$-axis, then another Type I crossing on the $x$-axis, and so on. Let $\eta_{1}, \eta_{2}, \ldots$ be the signs of the Type I crossings from left to right along the $x$-axis. Let $\left\{\varepsilon_{1}^{1}, \varepsilon_{1}^{2}\right\},\left\{\varepsilon_{2}^{1}, \varepsilon_{2}^{2}\right\}, \ldots$ be the signs of the pairs of Type II crossings from left to right. Proceeding in a fashion similar to that given on pages 300-303 in [14, we obtain that $p / q$ is given by the continued fraction

$$
p / q=\left[\eta_{1}, \varepsilon_{1}^{1}+\varepsilon_{1}^{2}, \eta_{2}, \varepsilon_{2}^{1}+\varepsilon_{2}^{2}, \ldots, \eta_{n_{y}}\right]=\eta_{1}+\frac{1}{\varepsilon_{1}^{1}+\varepsilon_{1}^{2}+\frac{1}{\eta_{2}+\cdots+\frac{1}{\eta_{n_{y}}}}}
$$

Note that if $K$ is Lissajous then it is rotationally symmetric with respect to the $x$-axis and each pair of Type II crossings has the same sign. In this case each $\varepsilon_{i}^{1}+\varepsilon_{i}^{2}$ can be replaced with $2 \varepsilon_{i}^{1}$. Using this formula, it is easy to determine the 2 -bridge knot given by a Fourier- $(1,1,2)$ representation with $n_{x}=2$. Hence, when we sample Lissajous and Fourier- $(1,1,2)$ knots with $n_{x}=2$, even if we obtain knots with hundreds of crossings, it is a simple matter to distinguish them.

Since every Lissajous knot with $n_{x}=2$ is 2-bridge, a good question is: What 2-bridge knots are Lissajous with $n_{x}=2$ ? As mentioned in the Introduction, every Lissajous knot is either strongly plus amphicheiral, or 2-periodic and linking its axis of rotation once. It is known that a 2-bridge knot cannot be strongly plus amphicheiral 4. It is also known (and will be shown below) that every 2-bridge knot is 2-periodic, but may or may not link its axis of rotation once. The following theorem makes it easy to identify which 2-bridge knots might be Lissajous.

Theorem 10. Let $K$ be a 2-bridge knot. Then the following are equivalent.

1) $K$ has a symmetry of period 2 with axis $A$ such that $A$ is disjoint from $K$ and $|l k(A, K)|=1$

2) $\Delta_{K}(t)$ is a square $\bmod 2$

3) $\Delta_{K}(t) \equiv 1 \bmod 2$.

Proof. As already mentioned in the introduction, it follows from a result of Murasugi (see [12]) that 1) implies 2) and clearly 3) implies 2). We must show that 2) implies both 1) and 3).

Suppose $K$ is a 2-bridge knot given by the pair of relatively prime integers $(p, q)$ with $p$ odd and $0<q<p$. There is a unique continued fraction expansion

$$
p / q=\left[2 a_{1},-2 a_{2}, \ldots,(-1)^{n+1} 2 a_{n}\right]
$$

where each of the partial quotients $(-1)^{i+1} 2 a_{i}$ is even. Corresponding to this expansion is a Seifert surface made from plumbing together twisted bands as shown in Figure 4. Notice that the Seifert surface, and hence $K$ is rotationally symmetric around the axis $A$. Thus every 2-bridge knot has a symmetry of period 2 with 


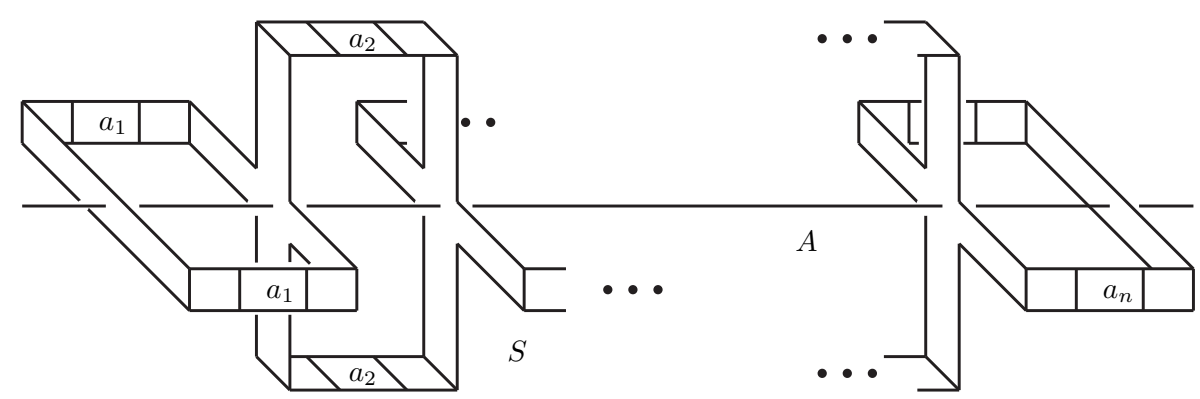

Figure 4: The Seifert surface $S$ and axis $A$ for the 2-bridge knot $K_{p / q}$. Each $a_{i}$ represents $a_{i}$ right handed half-twists in the band.

axis disjoint from the knot. However, the linking number of $A$ and $K$ need not be \pm 1 in general. From the plumbing picture we also see that the number of bands, $n$, must be even in order to get a knot. If $n$ is odd we obtain a 2-bridge link (of two components).

The axis $A$ meets the Seifert surface $S$ transversely in $n+1$ points and we may compute the linking number of $A$ and $K$ by counting the signed intersection points. Let $\epsilon_{i}$ be the sign of the intersection point that occurs between band $i$ and $i+1$. Let $\epsilon_{0}$ be the sign of the left-most intersection point in the figure and choose orientations so that $\epsilon_{0}=1$. It is easy to see that $\epsilon_{i+1}=\epsilon_{i}$ if $a_{i}$ is odd and $\epsilon_{i+1}=-\epsilon_{i}$ if $a_{i}$ is even. Thus the sequence $\left\{a_{1}, a_{2}, \ldots, a_{n}\right\}$ determines the sequence $\left\{\epsilon_{0}, \epsilon_{1}, \ldots, \epsilon_{n}\right\}$ which in turn determines the linking number between $A$ and $K$.

From the Seifert surface we may obtain the Seifert matrix $V$ and compute the Conway polynomial $\nabla(z)=$ $\operatorname{det}\left(t^{-1 / 2} V-t^{1 / 2} V^{T}\right)$. It is a straightforward calculation to show that

$$
\nabla_{K}(z)=\left(\begin{array}{ll}
1 & 0
\end{array}\right)\left(\begin{array}{cc}
-a_{1} z & 1 \\
1 & 0
\end{array}\right)\left(\begin{array}{cc}
-a_{2} z & 1 \\
1 & 0
\end{array}\right) \ldots\left(\begin{array}{cc}
-a_{n} z & 1 \\
1 & 0
\end{array}\right)\left(\begin{array}{l}
1 \\
0
\end{array}\right)
$$

(See page 207 of [3].)

If $n=2$ the Conway polynomial is $\nabla(z)=1+a_{1} a_{2} z^{2}$ and the Alexander polynomial $\Delta(t)=\nabla\left(t^{1 / 2}-t^{1 / 2}\right)=$ $a_{1} a_{2} t^{-1}+\left(1-2 a_{1} a_{2}\right)+a_{1} a_{2} t$. In general, an Alexander polynomial of the form

$$
\Delta(t)=b_{0}+b_{1}\left(t+t^{-1}\right)+b_{2}\left(t^{2}+t^{-2}\right)+\cdots+b_{m}\left(t^{m}+t^{-m}\right)
$$

is a square mod 2 if and only if $b_{2 k+1} \equiv 0 \bmod 2$ for $k=0,1, \ldots$ Thus $a_{1} a_{2} t^{-1}+\left(1-2 a_{1} a_{2}\right)+a_{1} a_{2} t$ is a square mod 2 if and only if at least one of $a_{1}$ or $a_{2}$ is even. But if this is the case it follows that $\sum \epsilon_{i}= \pm 1$. It is also true that $a_{1} a_{2} t^{-1}+\left(1-2 a_{1} a_{2}\right)+a_{1} a_{2} t$ is a square mod 2 if and only if it is equal to 1 mod 2 .

Suppose now that $n>2$ and that $K$ is a knot with $\Delta_{K}(t)$ a square mod 2. The first thing to show is that $a_{i}$ is even for at least one value of $i$. If $a_{i}$ were odd for every $i$, then replace each $a_{i}$ with -1 . This does not change any $a_{i} \bmod 2$, and hence does not change $\nabla_{K}(z) \bmod 2$ or $\Delta_{K}(t) \bmod 2$. But if $a_{i}=-1$ for all $i$, we can prove by induction on $n$ that

$$
\Delta(t)=1-t+t^{2}-t^{3}+\cdots+t^{n}
$$

and it follows that $\Delta(t)$ is not a square mod 2. Thus at least one $a_{i}$ is even. Replacing this $a_{i}$ with zero transforms $K$ into a knot $J$ with the same Alexander polynomial mod 2 but with a Seifert surface having two fewer bands. Proceeding by induction on the number of bands, we have that $\operatorname{lk}(J, A)= \pm 1$. But now because $a_{i}$ is even, $K$ must also link $A$ once. If we begin instead with the assumption that $\Delta_{K}(t) \equiv 1 \bmod$ 2 , the same argument will work. 


\begin{tabular}{|cc|cc|cc|cc|}
\hline$n_{y}$ & $\left|\mathcal{L}\left(2, n_{y}\right)\right|$ & $n_{y}$ & $\left|\mathcal{L}\left(2, n_{y}\right)\right|$ & $n_{y}$ & $\left|\mathcal{L}\left(2, n_{y}\right)\right|$ & $n_{y}$ & $\left|\mathcal{L}\left(2, n_{y}\right)\right|$ \\
\hline 3 & 3 & 29 & 645 & 55 & 1854 & 81 & 3761 \\
5 & 11 & 31 & 737 & 57 & 1727 & 83 & 5805 \\
7 & 28 & 33 & 533 & 59 & 2859 & 85 & 4654 \\
9 & 37 & 35 & 684 & 61 & 3062 & 87 & 4195 \\
11 & 78 & 37 & 1075 & 63 & 1946 & 89 & 6707 \\
13 & 109 & 39 & 772 & 65 & 2639 & 91 & 5647 \\
15 & 93 & 41 & 1339 & 67 & 3708 & 93 & 4805 \\
17 & 203 & 43 & 1473 & 69 & 2593 & 95 & 5892 \\
19 & 258 & 45 & 904 & 71 & 4191 & 97 & 7984 \\
21 & 195 & 47 & 1782 & 73 & 4433 & 99 & 5208 \\
23 & 390 & 49 & 1688 & 75 & 2584 & 101 & 8699 \\
25 & 390 & 51 & 1365 & 77 & 3933 & 103 & 9036 \\
27 & 387 & 53 & 2287 & 79 & 5248 & 105 & 4425 \\
\hline
\end{tabular}

Table 1: The number of distinct Lissajous knots with $n_{x}=2$ as a function of $n_{y}$.

\section{$5 \quad$ Sampling Lissajous and Fourier Knots}

Using the results of Sections 2 we are now in a position to efficiently sample Lissajous knots. In the case where $n_{x}=2$ we obtain 2-bridge knots and can take advantage of this to compare knots in our sample. For the more general case of Fourier knots, we have not carried out a complete analysis of the phase torus, a task that seems much more difficult. Hence, we have not attempted to rigorously sample Fourier knots, but instead have relied on two methods, either random sampling or an algorithm which first "draws" a bitmap image of the phase torus (as in Figure 3) and then picks one point from each "white" region. This latter approach is fraught with difficulty since, for example, some white regions may be smaller than a single pixel and be missed. Our samples naturally fall into four cases which we describe in turn in this section.

\subsection{Lissajous Knots with $2=n_{x}<n_{y}<n_{z}$}

We have determined all knots in $\mathcal{L}\left(2, n_{y}\right)$ for $3 \leq n_{y} \leq 105$. For a given value of $n_{y}$ we let $n_{z}$ run from $3 n_{y}+2$ to $7 n_{y}$. These values of $n_{z}$ are sufficient to guarantee that we obtain all possible knots in $\mathcal{L}\left(2, n_{y}\right)$. Since each of these knots is 2-bridge we were able to use Equation[11 to identify the associated pair $(p, q)$ and thus compare knots in the output. The total number of knots in $\mathcal{L}\left(2, n_{y}\right)$ is given in Table 1 for each value of $n_{y}$. It is interesting to compare these numbers with the upper bound given by Theorem 8 . Depending on $n_{y}$, the actual number of knots found is roughly between 5 and 10 per cent of the upper bound. The discrepancy is almost certainly due to the presence of huge numbers of unknots. The $x y$-projection of a Lissajous knot with $n_{x}=2$ and $n_{y}=99$ has $(2)(2)(99)-2-99=295$ crossings, and knots in $\mathcal{L}(2,99)$ have crossing numbers ranging from 5 to 293. Of course the bound of 78008 given by Theorem 8 for $n_{x}=2$ and $n_{y}=99$ is well below the upper bound of $2^{295}$ obtained by considering all possible crossing arrangements!

The total number of knots in Table 1 is 135061, far too many to describe one by one. However, in Tables 47 we list all knots in $\mathcal{L}\left(2, n_{y}\right)$, grouped by crossing number, for $3 \leq n_{y} \leq 15$. Several interesting things can be seen in these tables. The same knot often appears in $\mathcal{L}\left(2, n_{y}\right)$ for many different values of $n_{y}$. For example $K_{7 / 2}$ (which is the twist knot $5_{2}$ in [14]) appears in every column of Table 4. In fact, $K_{7 / 2} \in \mathcal{L}\left(2, n_{y}\right.$ ) for $3 \leq n_{y} \leq 105$. This is also true for $K_{9 / 2}$. The knot $K_{15 / 4}$ first appears for $n_{y}=3$, misses a few values of $n_{y}$, and then is contained in $\mathcal{L}\left(2, n_{y}\right)$ for $23 \leq n_{y} \leq 105$. Similar patterns hold for the other small-crossing knots suggesting that if $K \in \mathcal{L}\left(2, n_{y}\right)$ for some $n_{y}$ then there exists $N$ such that $K \in \mathcal{L}\left(2, n_{y}\right)$ for all $n_{y} \geq N$. A second observation is that several small crossings knots are already conspicuously absent. In particular, 
there are exactly four 8-crossings knots with Alexander polynomial congruent to 1 mod 2 (and hence possibly Lissajous). These are $K_{17 / 4}, K_{23 / 7}, K_{25 / 9}$ and $K_{31 / 12}$, only one of which, $K_{31 / 12}$, appears to be Lissajous. While Tables 40 display only a small fraction of our total sample, it is in fact true that the other three 8-crossing knots do not appear for any $n_{y}$ up to 105.

Question 1. Does there exist a 2-bridge knot $K$ with $\Delta_{K}(t) \equiv 1 \bmod 2$ that is not Lissajous (with or without one frequency equal to 2)? In particular, are any of the 8-crossing 2-bridge knots $K_{17 / 4}, K_{23 / 7}$ or $K_{25 / 9}$ Lissajous?

In Table 2 we list the numbers of 2-bridge knots, 2-bridge knots with Alexander polynomial congruent to 1 mod 2, and finally, the number of these that are Lissajous knots with $n_{x}=2$ and $3 \leq n_{y} \leq 105$. The table has entries for each crossing number from 3 to 16 . Very quickly we see that many 2-bridge knots with the required symmetry are not Lissajous, at least not with $n_{x}=2$ and $3 \leq n_{y} \leq 105$. It seems unlikely that choosing $n_{y}>105$ will yield more 2 -bridge knots in the 3-16 crossing range. On the other hand, perhaps letting the even frequency be more than 2 will yield more 2-bridge knots with small crossing number. We examine this further in Section 5.2

crossing number

\begin{tabular}{|c|cccccccccccccc|}
\hline & 3 & 4 & 5 & 6 & 7 & 8 & 9 & 10 & 11 & 12 & 13 & 14 & 15 & 16 \\
\hline 2-bridge & 1 & 1 & 2 & 3 & 7 & 12 & 24 & 45 & 91 & 176 & 352 & 693 & 1387 & 2752 \\
$\Delta(t) \equiv 1$ & 0 & 0 & 1 & 1 & 2 & 4 & 8 & 13 & 26 & 51 & 97 & 185 & 365 & 705 \\
$\mathcal{L}\left(2, n_{y}\right)$ & 0 & 0 & 1 & 1 & 2 & 1 & 3 & 4 & 8 & 5 & 9 & 7 & 15 & 15 \\
\hline
\end{tabular}

Table 2: The number of 2-bridge knots, 2-bridge knots with Alexander polynomial congruent to 1 mod 2, and the number of these that are Lissajous with $n_{x}=2$ and $3 \leq n_{y} \leq 105$, as a function of crossing number.

In Tables 89 we list all 2-bridge knots with crossings from 3 to 16 which are Lissajous knots with $n_{x}=2$ and $3 \leq n_{y} \leq 105$. Here the knot name, as defined in [6] and used in Knotscape [5] appears in the first column. Following that we give the 2-bridge defining fraction $p / q$ and then the frequency and phase shift data. For each knot, the given value of $n_{y}$ is minimal. However, since our search let $n_{z}$ run from $3 n_{y}+2$ to $7 n_{y}$, it might be possible for a given knot to be represented with a smaller value of $n_{z}$.

As a check against errors, we took all the 2-bridge knots in the data set that have Lissajous diagrams with less than 50 crossings (the built-in limit for Knotscape) and crossing number less than 17, and looked them up in the Knotscape table of knots in two different ways. First we converted their Lissajous diagrams to Dowker-Thistlethwaite code (the input format for Knotscape) and then used the "Locate in Table" feature. Next we converted the defining fraction $p / q$ into DT code and again used the "Locate in Table" routine. Happily, the results matched.

\subsection{Lissajous Knots with $2<n_{x}<n_{y}<n_{z}$}

Our goal in this section is to simply find as many Lissajous knots in the $3-16$ crossing range as we can. We may still use the results of Section 2 to efficiently sample Lissajous knots with all frequencies greater than 2 , but it is more difficult to tabulate the output. This is because even with relatively small frequencies, very large crossing number knots can result, and we can no longer use the classification of 2-bridge knots to sort them out. Therefore, we limited ourselves to producing diagrams with at most 49 crossings, the limit of what can be input to Knotscape. Assuming that $2<n_{x}<n_{y}$, and that $\operatorname{gcd}\left(n_{x}, n_{y}\right)=1$, we are left with the following $\left(n_{x}, n_{y}\right)$ pairs:

$$
\{(3,4),(3,5),(3,7),(3,8),(3,10),(4,5),(4,7),(5,6)\} .
$$


For each of these pairs we let $n_{z}$ run from $2 n_{x} n_{y}-n_{x}-n_{y}$ to $4 n_{x} n_{y}-n_{x}-n_{y}-1$, a range sufficient to produce all possible Lissajous knots. We obtained a total of 6352 knots of which Knotscape identified 1428 as unknots. The remaining 4924 knots fell into four categories:

1. knots identified as composites by Knotscape,

2. knots which Knotscape located in the Hoste-Thistlethwaite-Weeks table,

3. knots which Knotscape simplified to alternating projections with more than 16 crossings, and

4. knots which Knotscape simplified to nonalternating projections with more than 16 crossings.

In Table 3 and Tables 10 11 we list all knots in the first two categories. We note that while Knotscape can identify a knot as a composite, it identifies the summands only up to mirror image. In order to properly identify the composites in Table 3 we compared their Jones polynomials to the Jones polynomials of all possible composites using the given summands or their mirror images in all possible ways.

\begin{tabular}{|l|cccccc|}
\hline knot & $n_{x}$ & $n_{y}$ & $n_{z}$ & $\phi_{x}$ & $\phi_{y}$ & $\phi_{z}$ \\
\hline $3 a 1 \# 3 a 1$ & 3 & 4 & 23 & 0 & 0.25210 & 1.84229 \\
$3 a 1 \# \overline{3 a 1}$ & 3 & 5 & 29 & 0 & 0.23099 & 2.91059 \\
$5 a 1 \# 5 a 1$ & 3 & 7 & 50 & 0 & 0.50522 & 1.58916 \\
$5 a 1 \# 5 a 1$ & 3 & 5 & 29 & 0 & 0.26179 & 1.83259 \\
$6 a 1 \# 6 a 1$ & 4 & 5 & 37 & 0 & 0.18699 & 2.95459 \\
$6 a 3 \# 6 a 3$ & 3 & 8 & 47 & 0 & 0.23799 & 0.80919 \\
$6 a 3 \# \overline{6 a 3}$ & 3 & 5 & 29 & 0 & 0.29259 & 0.75459 \\
$3 a 1 \# 3 a 1 \# 5 a 1$ & 4 & 5 & 39 & 0 & 0.16064 & 2.19554 \\
$3 a 1 \# 3 a 1 \# \overline{5 a 1}$ & 4 & 7 & 55 & 0 & 0.13934 & 2.21684 \\
$3 a 1 \# 3 a 1 \# 8 a 2$ & 5 & 6 & 59 & 0 & 0.11116 & 2.40211 \\
$5 a 1 \# 5 a 1 \# \overline{5 a 1}$ & 4 & 7 & 55 & 0 & 0.15201 & 1.41878 \\
$6 a 3 \# 6 a 3 \# \overline{6 a 3}$ & 4 & 7 & 55 & 0 & 0.16468 & 0.62071 \\
$3 a 1 \# 3 a 1 \# 3 a 1 \# 3 a 1$ & 5 & 6 & 59 & 0 & 0.10149 & 1.78345 \\
\hline
\end{tabular}

Table 3: Small-crossing composite Lissajous knots. A bar over a knot name indicates mirror image. Knot names are as in Knotscape.

The third category cannot include knots in the Hoste-Thistlethwaite-Weeks table and we make no attempt to list them here. The fourth category might have included knots with 16 or less crossings that Knotscape simply failed to simplify correctly. To investigate this we first computed the Jones polynomial of each knot and eliminated knots whose Jones polynomial had a span of 17 or more. (Recall that the crossing number of a knot is bounded below by the span of the Jones polynomial.) This left a total of 78 knots. Of these, only 5 shared the same Jones polynomial with prime knots having less than 17 crossings and furthermore having an Alexander polynomial that is a square mod 2. In each of these five cases either the Alexander polynomial or the Kauffman 2-variable polynomial was sufficient to show that the knots did indeed have crossing numbers of 17 or more.

Thus, barring clerical errors, Table 3 and Tables 1011 provide a complete list of all Lissajous knots with $x$ and $y$ frequencies of $(3,4),(3,5),(3,7),(3,8),(3,10),(4,5),(4,7)$ or $(5,6)$ which are either composite, or prime with 16 or less crossings.

As mentioned in the introduction, there are exactly three prime knots with 12 or less crossings that are strongly plus amphicheiral: 10a103 (1099), 10a121 (10 123$)$, and 12a427. The knots 10a103 and 12a427 are Lissajous and are listed in Table 10. A natural question is, 
Question 2. Is the strongly plus amphicheiral knot 10a121 Lissajous?

The knot 10a121 is one member of a family of knots known as Turks Head knots. These knots are conjectured to not be Lissajous by Przytycki. See [13].

It is easy to see that every composite knot of the form $K \# \bar{K}$ is strongly plus amphicheiral while composites of the form $K \# K$ are 2-periodic and link their axis of rotation once. Several knots of this form appear in Table 3. Thus another good question is,

Question 3. Is every composite knot of the form $K \# K$ or $K \# \bar{K}$ Lissajous?

\subsection{Fourier- $(1,1,2)$ Knots with $2=n_{x}<n_{y}$}

Rather than trying to algorithmically choose one point in each region of the phase torus for a Fourier- $(1,1,2)$ knot, we chose instead to randomly sample points from the phase torus. Fixing $n_{x}=2, \phi_{x}=0$ and $A_{z, 1}=1$, we then let $n_{y}$ take on odd values from 3 to 99 . For each value of $n_{y}$ the remaining parameters were then chosen at random such that:

$$
\begin{gathered}
\phi_{y}=\frac{k}{7} \pi, k \in\{1,2,3,4,5,6\} \\
0<n_{z, 1}<n_{z, 2}<301 \\
0 \leq \phi_{z, 1} \leq \pi \\
0 \leq \phi_{z, 2} \leq 2 \pi \\
0 \leq A_{z, 2} \leq 2
\end{gathered}
$$

For each value of $n_{y}$, random sampling in batches of 10000 took place until no new knots were found. If a knot was produced that had already been found, the one with the lexicographically smallest set $\left\{n_{x}, n_{y}, n_{z, 1}, n_{z, 2}\right\}$ was kept. This tended to produce knots with fairly small values of $\left\{n_{x}, n_{y}, n_{z, 1}\right.$ but with $n_{z, 2}$ often in the hundreds. Furthermore, only knots with less than 17 crossings were kept in the sample.

After a modest amount of searching, we turned up all 2-bridge knots with 14 or less crossings, and nearly all 15 and 16-crossing ones as well. (We found 1386 out of 138715 -crossing knots and 2731 out of 2752 16-crossing knots.) We believe the following conjecture is reasonable.

Conjecture 11. Every 2-bridge knot can be expressed as a Fourier- $(1,1, k)$ knot with $n_{x}=2$ and $k \leq 2$.

Additional evidence for this conjecture is provided by the twist knots. The twist knot $T_{m}$, which is the 2-bridge knot $K_{\frac{2 m+1}{2}}$, is shown in Figure 5. The mirror image of $T_{m}$ is the twist knot $T_{-1-m}$. Thus it suffices to consider $m>1$. It is shown in [7] that $T_{m}$ is Lissajous if and only if $m \equiv 0 \bmod 4$ or $m \equiv 3$ mod 4. If this is not the case, the knot does not have the required symmetry to be Lissajous. However, in these cases, the following examples show that $K_{m}$ is a Fourier- $(1,1,2)$ knot. Thus all twist knots are Fourier- $(1,1, k)$ knots with $k \leq 2$.

Theorem 12. Twist knots which are not Lissajous may be expressed as Fourier knots as follows.

1. The twist knot $T_{4 n+1}$ can be expressed as the Fourier- $(1,1,2)$ knot with $n_{x}=2, \phi_{x}=0, n_{y}=8 n+3, \phi_{y}=$ $1 / 2, n_{z, 1}=2, \phi_{z, 1}=\pi / 4, n_{z, 2}=8 n+1, \phi_{z, 2}=\frac{8 n+1+(8 n+5) \pi}{2(8 n+3)}$ and $A_{z, 2}=1$ for all $n \geq 1$.

2. The twist knot $T_{2 n}$ can be expressed as the Fourier- $(1,1,2)$ knot with $n_{x}=2, \phi_{x}=0, n_{y}=2 n+1, \phi_{y}=$ $1 / 2, n_{z, 1}=2, \phi_{z, 1}=\pi / 4, n_{z, 2}=2 n+3, \phi_{z, 2}=\frac{2 n+3-3 \pi}{2(2 n+1)}$ and $A_{z, 2}=1$ for all $n \geq 1$. 
The proof is similar to the proof of Theorem 4 given in [7] and relies on very carefully determining the sign of each crossing in the diagram. The details are quite long and not particularly insightful. We leave this as a rather complicated exercise for the reader.

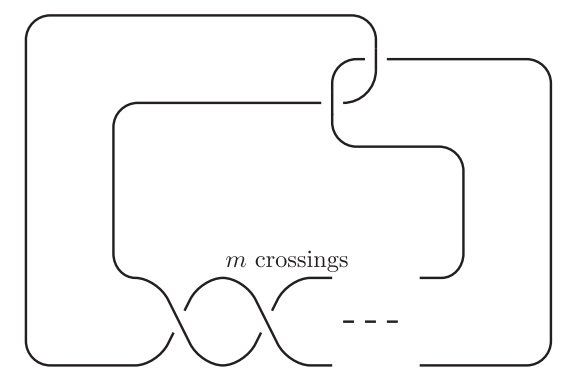

Figure 5: The twist knot $T_{m}$.

Our sample of all 2-bridge knots to 16 crossings expressed as Fourier- $(1,1,2)$ knots is too large to reproduce here. Instead, in Tables 12,13 we list all 2-bridge knots to 10 crossings with associated Fourier data. To generate this table we again undertook a random sample but this time sharply reduced the range of the parameters. In particular, we kept all amplitudes equal to one, set $\phi_{y}=\pi / 4$, and only allowed $z$-frequencies as large as 10. An interesting variation on Conjecture 11 would be to require that all amplitudes are 1. Knots appearing in Tables 12 13 which are known to be Lissajous are shown in boldface, while those that have Alexander polynomials congruent to $1 \bmod 2$, and hence might be Lissajous, are shown in italics.

\subsection{Fourier- $(1,1,2)$ Knots with $2<n_{x}<n_{y}$}

We made only a modest attempt to sample Fourier- $(1,1,2)$ knots with $x$ and $y$ frequencies greater than two. Rather than sampling at random as in Section [5.3, we now chose one sampling point from each region of the phase torus by first creating a bitmap image as in Figure 3 and then taking the centroid of each white region. Sometimes the centroid fell outside of the region and in this case an arbitrary point of the region was selected. Because of the large crossing numbers that result, and the consequent difficulty in identifying these knots, we again restricted our sample to $x$ and $y$ frequencies of $(3,4),(3,5),(3,7),(3,8),(3,10),(4,5),(4,7)$ or $(5,6)$. We further restricted the $z$-frequencies to be less than 15 and somewhat arbitrarily fixed all amplitudes at 1. Using Knotscape to identify the resulting knots, and keeping only knots with 16 or less crossings, we found several thousand prime knots. In Tables 14 16 we list all of these with 10 or less crossings which are not 2-bridge and thus listed in Tables 12,13, All knots through 9 crossings were found, and all but 20 alternating 10 -crossings knots were found. We suspect that limiting the $z$-frequencies to less than 15 is a severe restriction.

We did however, find all torus knots up to 16 crossings. It is shown by Kauffman in 9] that every torus knot is a Fourier- $(1,3,3)$ knot. Interestingly, we found that, up to 16 crossings, the torus knot $T_{p, q}$ can be represented by a Fourier- $(1,1,2)$ knot with $n_{x}=p$ and $n_{y}=q$. Table 18 lists these results. The data suggests the following conjectures (which we have verified for a large number of $(p, q)$ pairs, and hope to prove in a later paper).

Conjecture 13. The torus knot $T_{2, q}$ can be represented as a Fourier- $(1,1,2)$ knot with frequencies $n_{x}=$ $2, n_{y}=q, n_{z, 1}=2$ and $n_{z, 2}=q-2$ and phase shifts $\phi_{x}=0, \phi_{y}=\pi / 4, \phi_{z, 1}=\pi / 2$ and $\phi_{z, 2}=\pi / 4$. 
Conjecture 14. The torus knot $T_{p, q}$, with $0<p<q$, can be represented as a Fourier- $(1,1,2)$ knot with frequencies $n_{x}=p, n_{y}=q, n_{z, 1}=p$ and $n_{z, 2}=q-p$.

It would be interesting to undertake a large-scale sampling of Fourier- $(1,1,2)$ knots with $x$ and $y$ frequencies greater than two to see if every knot with 16 or less crossings turns up. Such a study might shed light on the following question,

Question 4. Is there a knot which cannot be expressed as a Fourier-(1,1,k) knot for $k \leq 2$ ?

\section{References}

[1] M. G. V. Bogle, J. E. Hearst, V. F. R. Jones, and L. Stoilov. Lissajous knots. J. Knot Theory Ramifications, 3(2):121-140, 1994.

[2] Gerhard Burde and Heiner Zieschang. Knots, volume 5 of de Gruyter Studies in Mathematics. Walter de Gruyter \& Co., Berlin, 2003.

[3] Peter Cromwell. Knots and Links. Cambridge University Press, 2004.

[4] Richard Hartley and Akio Kawauchi. Polynomials of amphicheiral knots. Math. Ann., 243(1):63-70, 1979.

[5] Jim Hoste and Morwen Thistlethwaite. Knotscape. http://www.math.utk.edu/ morwen 1998.

[6] Jim Hoste, Morwen Thistlethwaite, and Jeff Weeks. The first 1,701,936 knots. Math. Intelligencer, 20(4):33-48, 1998.

[7] Jim Hoste and Laura Zirbel. Lissajous knots with lissajous projections. arXiv: math.GT/0605632, 2006.

[8] Vaughan F. R. Jones and Józef H. Przytycki. Lissajous knots and and billiard knots. Banach Center Publications, (42):145-163, 1998.

[9] Louis H. Kauffman. Fourier Knots. arXiv: q-alg/9711013

[10] Christoph Lamm. Fourier knots. Preprint.

[11] Christoph Lamm. There are infinitely many lissajous knots. Manuscripta Math., 93:29-37, 1996.

[12] Kunio Murasugi. On periodic knots. Comment. Math. Helv., 46:162-174, 1971.

[13] Jozef Przytycki. Symmetric knots and billiard knots. arXiv: math.GT/0405151

[14] Dale Rolfsen. Knots and links, volume 7 of Mathematics Lecture Series. Publish or Perish Inc., Houston, TX, 1990. Corrected reprint of the 1976 original. 


\section{Tables of Lissajous and Fourier Knots}

\begin{tabular}{|l|c|c|c|c|c|c|c|}
\hline & $\mathcal{L}(2,3)$ & $\mathcal{L}(2,5)$ & $\mathcal{L}(2,7)$ & $\mathcal{L}(2,9)$ & $\mathcal{L}(2,11)$ & $\mathcal{L}(2,13)$ & $\mathcal{L}(2,15)$ \\
\hline cr & $p / q$ & $p / q$ & $p / q$ & $p / q$ & $p / q$ & $p / q$ & $p / q$ \\
\hline 5 & $7 / 2$ & $7 / 2$ & $7 / 2$ & $7 / 2$ & $7 / 2$ & $7 / 2$ & $7 / 2$ \\
\hline 6 & $9 / 2$ & $9 / 2$ & $9 / 2$ & $9 / 2$ & $9 / 2$ & $9 / 2$ & $9 / 2$ \\
\hline 7 & $15 / 4$ & $17 / 5$ & $15 / 4$ & $17 / 5$ & $15 / 4$ & $15 / 4$ & $17 / 5$ \\
7 & & & $17 / 5$ & & $17 / 5$ & $17 / 5$ & \\
\hline 8 & & & & & $31 / 12$ & & \\
\hline 9 & & & $15 / 2$ & $31 / 7$ & $15 / 2$ & $15 / 2$ & $15 / 2$ \\
9 & & & $31 / 7$ & & $31 / 7$ & $31 / 7$ & $31 / 7$ \\
9 & & & & & $31 / 11$ & & \\
\hline 10 & & $17 / 2$ & $17 / 2$ & $17 / 2$ & $55 / 12$ & $17 / 2$ & $55 / 12$ \\
10 & & $49 / 20$ & $49 / 20$ & $57 / 16$ & $57 / 16$ & $49 / 20$ & $57 / 16$ \\
10 & & $57 / 16$ & $55 / 12$ & & & $57 / 16$ & \\
10 & & & $57 / 16$ & & & & \\
\hline 11 & & $65 / 14$ & $73 / 16$ & $49 / 9$ & $49 / 9$ & $41 / 13$ & $49 / 9$ \\
11 & & $73 / 16$ & & $65 / 14$ & $73 / 16$ & $49 / 9$ & $73 / 16$ \\
11 & & $97 / 26$ & & $73 / 16$ & & $71 / 20$ & \\
11 & & & & & & $73 / 16$ & \\
11 & & & & & & $97 / 26$ & \\
\hline 12 & & $121 / 32$ & $169 / 50$ & $167 / 46$ & $121 / 32$ & $167 / 46$ & $169 / 50$ \\
12 & & & & & $169 / 50$ & & \\
\hline 13 & & $209 / 56$ & $239 / 71$ & & $23 / 2$ & $71 / 11$ & $23 / 2$ \\
13 & & & & & $71 / 11$ & $209 / 56$ & $71 / 11$ \\
\hline 14 & & & $25 / 2$ & $25 / 2$ & $407 / 119$ & $25 / 2$ & $407 / 119$ \\
14 & & & $89 / 36$ & $89 / 36$ & & $409 / 121$ & \\
14 & & & $289 / 118$ & $289 / 118$ & & & \\
14 & & & & $409 / 121$ & & & \\
\hline 15 & & & $151 / 20$ & $441 / 101$ & $151 / 20$ & $97 / 13$ & $97 / 13$ \\
15 & & & $319 / 144$ & $463 / 130$ & $319 / 144$ & $361 / 78$ & $463 / 130$ \\
15 & & & $359 / 82$ & & $359 / 82$ & $463 / 130$ & \\
15 & & & $463 / 130$ & & $433 / 122$ & & \\
15 & & & & & $447 / 98$ & & \\
15 & & & & & $463 / 130$ & & $975 / 274$ \\
\hline 16 & & & $529 / 114$ & $593 / 130$ & $593 / 130$ & $529 / 114$ & $593 / 130$ \\
16 & & & $593 / 130$ & & $817 / 239$ & $559 / 122$ & $817 / 239$ \\
16 & & & $777 / 208$ & & & $593 / 130$ & \\
16 & & & & & & $815 / 237$ & \\
\hline 17 & & & $975 / 274$ & & $703 / 131$ & $1353 / 380$ & $31 / 2$ \\
17 & & & $983 / 260$ & & $1321 / 288$ & & $127 / 15$ \\
17 & & & $1351 / 362$ & & & & $983 / 260$ \\
17 & & & & & & & \\
\hline
\end{tabular}

Table 4: The sets $L\left(2, n_{y}\right)$ for $3 \leq n_{y} \leq 15$ given by crossing number and 2-bridge fraction $p / q$. 


\begin{tabular}{|c|c|c|c|c|c|c|c|}
\hline & $\mathcal{L}(2,3)$ & $\mathcal{L}(2,5)$ & $\mathcal{L}(2,7)$ & $\mathcal{L}(2,9)$ & $\mathcal{L}(2,11)$ & $\mathcal{L}(2,13)$ & $\mathcal{L}(2,15)$ \\
\hline $\mathrm{cr}$ & $p / q$ & $p / q$ & $p / q$ & $p / q$ & $p / q$ & $p / q$ & $p / q$ \\
\hline 18 & & & $1681 / 450$ & $33 / 2$ & $33 / 2$ & $1489 / 337$ & $241 / 32$ \\
\hline 18 & & & & $129 / 52$ & $129 / 52$ & & $1487 / 335$ \\
\hline 18 & & & & $529 / 214$ & $241 / 32$ & & \\
\hline 18 & & & & $1681 / 696$ & $529 / 214$ & & \\
\hline 18 & & & & $2321 / 622$ & $1681 / 696$ & & \\
\hline 19 & & & $2911 / 780$ & $273 / 32$ & $4063 / 1202$ & $273 / 32$ & \\
\hline 19 & & & & $673 / 78$ & & $673 / 78$ & \\
\hline 19 & & & & $1961 / 800$ & & $1025 / 161$ & \\
\hline 19 & & & & $2001 / 898$ & & $1961 / 800$ & \\
\hline 19 & & & & $3329 / 989$ & & $2001 / 898$ & \\
\hline 19 & & & & & & $4015 / 1106$ & \\
\hline 20 & & & & $3761 / 1056$ & $1279 / 282$ & $3409 / 744$ & $2481 / 559$ \\
\hline 20 & & & & & $3535 / 996$ & $3521 / 992$ & $3631 / 796$ \\
\hline 20 & & & & & $3761 / 1056$ & $3761 / 1056$ & $3761 / 1056$ \\
\hline 20 & & & & & $5681 / 1661$ & & \\
\hline 21 & & & & $4297 / 926$ & $4817 / 1056$ & $4817 / 1056$ & $1407 / 191$ \\
\hline 21 & & & & $4305 / 944$ & $6143 / 1407$ & $9833 / 2909$ & $4817 / 1056$ \\
\hline 21 & & & & $4817 / 1056$ & & & \\
\hline 22 & & & & $7921 / 2224$ & $41 / 2$ & $41 / 2$ & $10737 / 2354$ \\
\hline 22 & & & & $7985 / 2112$ & $169 / 68$ & $169 / 68$ & \\
\hline 22 & & & & $10865 / 2912$ & $769 / 310$ & $769 / 310$ & \\
\hline 22 & & & & & $3073 / 1272$ & $3073 / 1272$ & \\
\hline 22 & & & & & $9801 / 4058$ & $9801 / 4058$ & \\
\hline 22 & & & & & $11257 / 3102$ & $10471 / 3098$ & \\
\hline 22 & & & & & & $11441 / 3327$ & \\
\hline 23 & & & & $18817 / 5042$ & $415 / 36$ & $\begin{array}{l}2129 / 282 \\
\end{array}$ & $415 / 36$ \\
\hline 23 & & & & & $1223 / 146$ & $9793 / 1825$ & $1223 / 146$ \\
\hline 23 & & & & & $3487 / 1424$ & & $3487 / 1424$ \\
\hline 23 & & & & & $11759 / 4802$ & & $11759 / 4802$ \\
\hline 23 & & & & & $11999 / 4900$ & & $11999 / 4900$ \\
\hline 23 & & & & & $18663 / 4996$ & & \\
\hline 24 & & & & $23409 / 6272$ & $2425 / 322$ & $33433 / 9892$ & $21009 / 4733$ \\
\hline 24 & & & & & $6041 / 800$ & & $31759 / 6924$ \\
\hline 24 & & & & & $12769 / 5762$ & & \\
\hline 24 & & & & & $14369 / 3282$ & & \\
\hline 24 & & & & & $32593 / 8738$ & & \\
\hline 25 & & & & $40545 / 10864$ & $10359 / 2284$ & $10377 / 2288$ & $14279 / 2243$ \\
\hline 25 & & & & & $16511 / 3554$ & $28743 / 8098$ & $30551 / 8578$ \\
\hline 25 & & & & & $18583 / 4000$ & $30551 / 8578$ & \\
\hline 25 & & & & & $23543 / 5086$ & $55801 / 14954$ & \\
\hline 25 & & & & & $30551 / 8578$ & & \\
\hline 25 & & & & & $46367 / 13775$ & & \\
\hline
\end{tabular}

Table 5 : The sets $L\left(2, n_{y}\right)$ for $3 \leq n_{y} \leq 15$ given by crossing number and 2 -bridge fraction $p / q$. 


\begin{tabular}{|c|c|c|c|c|c|c|c|}
\hline & $\mathcal{L}(2,3)$ & $\mathcal{L}(2,5)$ & $\mathcal{L}(2,7)$ & $\mathcal{L}(2,9)$ & $\mathcal{L}(2,11)$ & $\mathcal{L}(2,13)$ & $\mathcal{L}(2,15)$ \\
\hline $\mathrm{cr}$ & $p / q$ & $p / q$ & $p / q$ & $p / q$ & $p / q$ & $p / q$ & $p / q$ \\
\hline 26 & & & & & $34905 / 7522$ & $49 / 2$ & $49 / 2$ \\
\hline 26 & & & & & $34969 / 7666$ & $209 / 84$ & $209 / 84$ \\
\hline 26 & & & & & $39129 / 8578$ & $1009 / 406$ & $1009 / 406$ \\
\hline 26 & & & & & $51193 / 14384$ & $4465 / 1848$ & $4465 / 1848$ \\
\hline 26 & & & & & & $17921 / 7418$ & $17921 / 7418$ \\
\hline 26 & & & & & & $39129 / 8578$ & $39129 / 8578$ \\
\hline 26 & & & & & & $57121 / 23660$ & $57121 / 23660$ \\
\hline 26 & & & & & & $80305 / 23857$ & $97681 / 28898$ \\
\hline 27 & & & & & $64343 / 18066$ & $601 / 48$ & $3871 / 514$ \\
\hline 27 & & & & & $64351 / 18068$ & $1793 / 142$ & \\
\hline 27 & & & & & $64863 / 17156$ & $6409 / 2592$ & \\
\hline 27 & & & & & $87031 / 23298$ & $21401 / 8738$ & \\
\hline 27 & & & & & & $69121 / 28222$ & \\
\hline 27 & & & & & & $69361 / 28320$ & \\
\hline 27 & & & & & & $85561 / 19597$ & \\
\hline 27 & & & & & & $91193 / 25584$ & \\
\hline 28 & & & & & $108241 / 28622$ & $4385 / 514$ & $162521 / 48279$ \\
\hline 28 & & & & & $108657 / 28732$ & $10817 / 1268$ & \\
\hline 28 & & & & & $151313 / 40544$ & $26937 / 3122$ & \\
\hline 28 & & & & & & $78489 / 32020$ & \\
\hline 28 & & & & & & $80089 / 35940$ & \\
\hline 28 & & & & & & $151697 / 44882$ & \\
\hline 29 & & & & & $188175 / 50374$ & $21441 / 2840$ & $136399 / 25419$ \\
\hline 29 & & & & & $188287 / 50404$ & $50969 / 11642$ & \\
\hline 29 & & & & & $262087 / 70226$ & $81025 / 36576$ & \\
\hline 29 & & & & & & $91193 / 20830$ & \\
\hline 29 & & & & & & $115561 / 26396$ & \\
\hline 29 & & & & & & $259969 / 69676$ & \\
\hline 30 & & & & & $326041 / 87362$ & $84041 / 18530$ & $57 / 2$ \\
\hline 30 & & & & & & $134689 / 28992$ & $249 / 100$ \\
\hline 30 & & & & & & $151553 / 32622$ & $1249 / 502$ \\
\hline 30 & & & & & & $191561 / 41968$ & $5857 / 2424$ \\
\hline 30 & & & & & & $248169 / 69680$ & $26041 / 10778$ \\
\hline 30 & & & & & & $453929 / 121630$ & $104441 / 43260$ \\
\hline 30 & & & & & & & $248169 / 69680$ \\
\hline 30 & & & & & & & $332929 / 137902$ \\
\hline 30 & & & & & & & $448689 / 120112$ \\
\hline
\end{tabular}

Table 6: The sets $L\left(2, n_{y}\right)$ for $3 \leq n_{y} \leq 15$ given by crossing number and 2-bridge fraction $p / q$. 


\begin{tabular}{|c|c|c|c|c|c|c|c|}
\hline & $\overline{\mathcal{L}(2,3)}$ & $\overline{\mathcal{L}(2,5)}$ & $\overline{\mathcal{L}(2,7)}$ & $\overline{\mathcal{L}(2,9)}$ & $\mathcal{L}(2,11)$ & $\mathcal{L}(2,13)$ & $\mathcal{L}(2,15)$ \\
\hline $\mathrm{cr}$ & $p / q$ & $p / q$ & $p / q$ & $p / q$ & $p / q$ & $p / q$ & $p / q$ \\
\hline 31 & & & & & $564719 / 151316$ & $283537 / 61102$ & $807 / 52$ \\
\hline 31 & & & & & & $284049 / 62270$ & $2599 / 210$ \\
\hline 31 & & & & & & $284057 / 62272$ & $9215 / 3728$ \\
\hline 31 & & & & & & $317849 / 69680$ & $39159 / 15842$ \\
\hline 31 & & & & & & $415929 / 116866$ & $127399 / 51540$ \\
\hline 31 & & & & & & $645809 / 191861$ & $317849 / 69680$ \\
\hline 31 & & & & & & & $400895 / 166464$ \\
\hline 31 & & & & & & & $402287 / 164258$ \\
\hline 32 & & & & & & $522665 / 146752$ & $1118489 / 332261$ \\
\hline 32 & & & & & & $522729 / 146768$ & \\
\hline 32 & & & & & & $526889 / 139360$ & \\
\hline 32 & & & & & & $697745 / 186784$ & \\
\hline 33 & & & & & & $864945 / 228716$ & $38951 / 5172$ \\
\hline 33 & & & & & & $872153 / 230622$ & $97015 / 12882$ \\
\hline 33 & & & & & & $882809 / 233440$ & $241791 / 32020$ \\
\hline 33 & & & & & & $1216977 / 326170$ & $511079 / 230624$ \\
\hline 33 & & & & & & & $511119 / 230642$ \\
\hline 33 & & & & & & & $575119 / 131362$ \\
\hline 33 & & & & & & & $1191711 / 272951$ \\
\hline 34 & & & & & & $1509537 / 404098$ & $2090425 / 559602$ \\
\hline 34 & & & & & & $1515361 / 407460$ & \\
\hline 34 & & & & & & $2107561 / 564720$ & \\
\hline 35 & & & & & & $2621905 / 702714$ & $2015903 / 566018$ \\
\hline 35 & & & & & & $2622017 / 702520$ & \\
\hline 35 & & & & & & $3650401 / 978122$ & \\
\hline 36 & & & & & & $4541161 / 1216800$ & $2303201 / 496338$ \\
\hline 36 & & & & & & & $2307361 / 505826$ \\
\hline 36 & & & & & & & $2307425 / 505842$ \\
\hline 36 & & & & & & & $2581921 / 566018$ \\
\hline 36 & & & & & & & $6322681 / 1694162$ \\
\hline 37 & & & & & & $7865521 / 2107560$ & $4245663 / 1192082$ \\
\hline 37 & & & & & & & $4246175 / 1192226$ \\
\hline 37 & & & & & & & $4246183 / 1192212$ \\
\hline 37 & & & & & & & $4279975 / 1132036$ \\
\hline 37 & & & & & & & $8994959 / 2672279$ \\
\hline 38 & & & & & & & $9748249 / 2609584$ \\
\hline$\overline{40}$ & & & & & & & $21077281 / 5642338$ \\
\hline 40 & & & & & & & $21089825 / 5645698$ \\
\hline 40 & & & & & & & $29354521 / 7865520$ \\
\hline 41 & & & & & & & $50843527 / 13623482$ \\
\hline 42 & & & & & & & $63250209 / 16947842$ \\
\hline 43 & & & & & & & $109552575 / 29354524$ \\
\hline
\end{tabular}

Table 7: The sets $L\left(2, n_{y}\right)$ for $3 \leq n_{y} \leq 15$ given by crossing number and 2-bridge fraction $p / q$. 


\begin{tabular}{|c|c|c|c|c|c|c|c|}
\hline knot & $\mathrm{p} / \mathrm{q}$ & $n_{x}$ & $n_{y}$ & $n_{z}$ & $\phi_{x}$ & $\phi_{y}$ & $\phi_{z}$ \\
\hline $5 \mathrm{a} 1$ & $7 / 2$ & 2 & 3 & 11 & 0 & 0.56099 & 2.58059 \\
\hline $6 \mathrm{a} 3$ & $9 / 2$ & 2 & 3 & 11 & 0 & 0.67319 & 0.89759 \\
\hline $7 \mathrm{a} 3$ & $17 / 5$ & 2 & 5 & 17 & 0 & 0.49979 & 2.64179 \\
\hline $7 a 6$ & $15 / 4$ & 2 & 3 & 11 & 0 & 0.78539 & 2.35619 \\
\hline $8 \mathrm{a} 1$ & $31 / 12$ & 2 & 11 & 41 & 0 & 0.39269 & 2.74889 \\
\hline $9 \mathrm{a} 8$ & $31 / 11$ & 2 & 11 & 41 & 0 & 0.48332 & 1.08747 \\
\hline $9 \mathrm{a} 27$ & $15 / 2$ & 2 & 7 & 25 & 0 & 0.49087 & 1.07992 \\
\hline 9a33 & $31 / 7$ & 2 & 7 & 23 & 0 & 0.47123 & 2.67035 \\
\hline $10 \mathrm{a} 23$ & $49 / 20$ & 2 & 5 & 17 & 0 & 0.71399 & 0.85679 \\
\hline $10 \mathrm{a} 63$ & $55 / 12$ & 2 & 7 & 25 & 0 & 0.44178 & 2.69980 \\
\hline 10a69 & $57 / 16$ & 2 & 5 & 19 & 0 & 0.58904 & 2.55254 \\
\hline $10 \mathrm{a} 75$ & $17 / 2$ & 2 & 5 & 17 & 0 & 0.57119 & 0.99959 \\
\hline 11a91 & $129 / 49$ & 2 & 41 & 153 & 0 & 0.34816 & 2.79342 \\
\hline 11a140 & $65 / 17$ & 2 & 17 & 63 & 0 & 0.47123 & 1.09955 \\
\hline 11a192 & $97 / 26$ & 2 & 5 & 17 & 0 & 0.64259 & 2.49899 \\
\hline 11a210 & $73 / 16$ & 2 & 5 & 19 & 0 & 0.65449 & 0.91629 \\
\hline 11a226 & $71 / 20$ & 2 & 13 & 49 & 0 & 0.45603 & 1.11475 \\
\hline $11 \mathrm{a} 246$ & $41 / 13$ & 2 & 13 & 47 & 0 & 0.47123 & 1.09955 \\
\hline 11a333 & $65 / 14$ & 2 & 5 & 19 & 0 & 0.78539 & 0.78539 \\
\hline 11a334 & $49 / 9$ & 2 & 9 & 29 & 0 & 0.45470 & 2.68688 \\
\hline 12a38 & $71 / 28$ & 2 & 25 & 89 & 0 & 0.41336 & 1.15742 \\
\hline 12a257 & $191 / 74$ & 2 & 17 & 63 & 0 & 0.37306 & 2.76852 \\
\hline 12a715 & $169 / 50$ & 2 & 7 & 25 & 0 & 0.53996 & 2.60163 \\
\hline 12a729 & $167 / 46$ & 2 & 9 & 31 & 0 & 0.43196 & 2.70962 \\
\hline 12a1034 & $121 / 32$ & 2 & 5 & 19 & 0 & 0.71994 & 2.42164 \\
\hline 13a640 & $55 / 19$ & 2 & 19 & 65 & 0 & 0.44879 & 1.12199 \\
\hline 13a1884 & $289 / 80$ & 2 & 25 & 93 & 0 & 0.35941 & 2.78217 \\
\hline $13 \mathrm{a} 2683$ & $287 / 79$ & 2 & 63 & 235 & 0 & 0.34262 & 2.79896 \\
\hline $13 \mathrm{a} 2760$ & $239 / 71$ & 2 & 7 & 23 & 0 & 0.57595 & 2.56563 \\
\hline 13a3143 & $23 / 2$ & 2 & 11 & 37 & 0 & 0.45814 & 1.11264 \\
\hline $13 \mathrm{a} 3896$ & $111 / 23$ & 2 & 23 & 85 & 0 & 0.46542 & 1.10537 \\
\hline 13a4304 & $209 / 56$ & 2 & 5 & 17 & 0 & 0.78539 & 2.35619 \\
\hline $13 \mathrm{a} 4570$ & $79 / 19$ & 2 & 19 & 69 & 0 & 0.46409 & 1.10669 \\
\hline $13 \mathrm{a} 4822$ & $71 / 11$ & 2 & 11 & 35 & 0 & 0.44392 & 2.69767 \\
\hline
\end{tabular}

Table 8: All Lissajous knots with frequencies $n_{x}=2,3 \leq n_{y} \leq 105$ and with less than 14 crossings. Knot names are as in Knotscape. 


\begin{tabular}{|l|c|cccccc|}
\hline knot & $\mathrm{p} / \mathrm{q}$ & $n_{x}$ & $n_{y}$ & $n_{z}$ & $\phi_{x}$ & $\phi_{y}$ & $\phi_{z}$ \\
\hline $14 \mathrm{a} 2651$ & $89 / 36$ & 2 & 7 & 23 & 0 & 0.62831 & 0.94247 \\
$14 \mathrm{a} 6166$ & $289 / 118$ & 2 & 7 & 23 & 0 & 0.73303 & 0.83775 \\
$14 \mathrm{a} 12186$ & $407 / 119$ & 2 & 11 & 37 & 0 & 0.42542 & 2.71616 \\
$14 \mathrm{a} 12212$ & $409 / 121$ & 2 & 9 & 31 & 0 & 0.51050 & 2.63108 \\
$14 \mathrm{a} 12308$ & $103 / 12$ & 2 & 57 & 215 & 0 & 0.36382 & 2.77776 \\
$14 \mathrm{a} 12652$ & $127 / 28$ & 2 & 29 & 103 & 0 & 0.40459 & 1.16619 \\
$14 \mathrm{a} 12741$ & $25 / 2$ & 2 & 7 & 23 & 0 & 0.52359 & 1.04719 \\
\hline $15 \mathrm{a} 21965$ & $113 / 29$ & 2 & 29 & 99 & 0 & 0.44178 & 1.12900 \\
$15 \mathrm{a} 25723$ & $745 / 288$ & 2 & 19 & 71 & 0 & 0.40142 & 2.74016 \\
$15 \mathrm{a} 32142$ & $319 / 144$ & 2 & 7 & 25 & 0 & 0.78539 & 0.78539 \\
$15 \mathrm{a} 44612$ & $359 / 82$ & 2 & 7 & 25 & 0 & 0.68722 & 0.88357 \\
$15 \mathrm{a} 46260$ & $361 / 78$ & 2 & 13 & 49 & 0 & 0.50670 & 1.06408 \\
$15 \mathrm{a} 50643$ & $447 / 98$ & 2 & 11 & 39 & 0 & 0.40840 & 2.73318 \\
$15 \mathrm{a} 50772$ & $433 / 122$ & 2 & 11 & 41 & 0 & 0.45311 & 2.68847 \\
$15 \mathrm{a} 51438$ & $463 / 130$ & 2 & 7 & 27 & 0 & 0.60059 & 2.54099 \\
$15 \mathrm{a} 52567$ & $151 / 20$ & 2 & 7 & 25 & 0 & 0.58904 & 0.98174 \\
$15 \mathrm{a} 54893$ & $65 / 21$ & 2 & 21 & 71 & 0 & 0.44392 & 1.12687 \\
$15 \mathrm{a} 71359$ & $505 / 109$ & 2 & 85 & 317 & 0 & 0.33994 & 2.80164 \\
$15 \mathrm{a} 71603$ & $169 / 29$ & 2 & 29 & 107 & 0 & 0.46199 & 1.10879 \\
$15 \mathrm{a} 76044$ & $441 / 101$ & 2 & 9 & 29 & 0 & 0.53737 & 2.60421 \\
$15 \mathrm{a} 78853$ & $129 / 25$ & 2 & 25 & 91 & 0 & 0.46040 & 1.11039 \\
$15 \mathrm{a} 84772$ & $97 / 13$ & 2 & 13 & 41 & 0 & 0.43633 & 2.70526 \\
\hline $16 \mathrm{a} 7016$ & $111 / 44$ & 2 & 39 & 131 & 0 & 0.40655 & 1.16423 \\
$16 \mathrm{a} 57423$ & $431 / 170$ & 2 & 39 & 139 & 0 & 0.40593 & 1.16486 \\
$16 \mathrm{a} 135506$ & $1103 / 456$ & 2 & 23 & 85 & 0 & 0.36361 & 2.77798 \\
$16 \mathrm{a} 219884$ & $961 / 208$ & 2 & 93 & 347 & 0 & 0.33914 & 2.80244 \\
$16 \mathrm{a} 221291$ & $777 / 208$ & 2 & 7 & 25 & 0 & 0.63813 & 2.50345 \\
$16 \mathrm{a} 221836$ & $783 / 220$ & 2 & 41 & 155 & 0 & 0.37667 & 2.76492 \\
$16 \mathrm{a} 224238$ & $791 / 212$ & 2 & 17 & 65 & 0 & 0.44058 & 2.70100 \\
$16 \mathrm{a} 225074$ & $593 / 130$ & 2 & 7 & 27 & 0 & 0.64679 & 0.92399 \\
$16 \mathrm{a} 228722$ & $559 / 122$ & 2 & 13 & 47 & 0 & 0.39269 & 2.74889 \\
$16 \mathrm{a} 229409$ & $577 / 162$ & 2 & 21 & 79 & 0 & 0.43982 & 1.13097 \\
$16 \mathrm{a} 249132$ & $817 / 239$ & 2 & 11 & 37 & 0 & 0.49087 & 2.65071 \\
$16 \mathrm{a} 249195$ & $815 / 237$ & 2 & 13 & 43 & 0 & 0.42074 & 2.72084 \\
$16 \mathrm{a} 252419$ & $385 / 114$ & 2 & 43 & 153 & 0 & 0.40071 & 1.17008 \\
$16 \mathrm{a} 252465$ & $399 / 110$ & 2 & 47 & 177 & 0 & 0.37166 & 2.76993 \\
$16 \mathrm{a} 333209$ & $529 / 114$ & 2 & 7 & 27 & 0 & 0.73919 & 0.83159 \\
\hline
\end{tabular}

Table 9: All Lissajous knots with frequencies $n_{x}=2,3 \leq n_{y} \leq 105$ and with $14-16$ crossings. Knot names are as in Knotscape. 


\begin{tabular}{|l|cccccc|}
\hline knot & $n_{x}$ & $n_{y}$ & $n_{z}$ & $\phi_{x}$ & $\phi_{y}$ & $\phi_{z}$ \\
\hline 5a1 & 3 & 4 & 29 & 0 & 0.49186 & 1.60252 \\
\hline 6a3 & 3 & 4 & 29 & 0 & 0.39666 & 1.69772 \\
\hline 7a6 & 3 & 7 & 68 & 0 & 0.52359 & 2.61799 \\
\hline 8a2 & 3 & 4 & 23 & 0 & 0.29088 & 2.85070 \\
8n2 & 3 & 4 & 37 & 0 & 0.49805 & 2.64353 \\
\hline 9a25 & 3 & 5 & 28 & 0 & 0.26973 & 1.82466 \\
\hline 10a20 & 3 & 4 & 23 & 0 & 0.32967 & 0.71752 \\
10a73 & 3 & 7 & 40 & 0 & 0.23394 & 2.90764 \\
10a89 & 3 & 4 & 29 & 0 & 0.36493 & 0.68226 \\
10a103 & 3 & 5 & 29 & 0 & 0.41579 & 2.72579 \\
10n28 & 3 & 7 & 40 & 0 & 0.21166 & 1.88272 \\
\hline 11n50 & 4 & 5 & 57 & 0 & 0.36736 & 1.98883 \\
11n151 & 4 & 7 & 69 & 0 & 0.24802 & 1.32277 \\
\hline 12a426 & 3 & 10 & 83 & 0 & 0.45603 & 2.68555 \\
12a427 & 3 & 5 & 29 & 0 & 0.32339 & 2.81819 \\
12a448 & 3 & 5 & 28 & 0 & 0.30146 & 0.74573 \\
12a868 & 3 & 5 & 26 & 0 & 0.28713 & 1.80726 \\
12a1164 & 4 & 5 & 61 & 0 & 0.34509 & 2.01109 \\
12n133 & 3 & 4 & 23 & 0 & 0.36845 & 1.72593 \\
12n293 & 3 & 10 & 59 & 0 & 0.28077 & 1.81362 \\
12n322 & 4 & 5 & 37 & 0 & 0.16829 & 2.18789 \\
12n483 & 5 & 6 & 113 & 0 & 0.35111 & 2.16215 \\
\hline 13a2233 & 3 & 8 & 67 & 0 & 0.48171 & 2.65988 \\
13a4774 & 3 & 4 & 23 & 0 & 0.40724 & 2.73434 \\
13n1405 & 4 & 5 & 51 & 0 & 0.35062 & 2.00557 \\
13n1734 & 3 & 10 & 103 & 0 & 0.56066 & 1.53372 \\
13n3594 & 3 & 4 & 23 & 0 & 0.44602 & 0.60116 \\
\hline
\end{tabular}

Table 10: Small-crossing Lissajous knots with all frequencies greater than 2. Only three knots, $5 a 1,6 a 3$ and $7 a 6$ are 2-bridge and appear in Table 8 , Knot names are as in Knotscape. 


\begin{tabular}{|l|cccccc|}
\hline knot & $n_{x}$ & $n_{y}$ & $n_{z}$ & $\phi_{x}$ & $\phi_{y}$ & $\phi_{z}$ \\
\hline $14 \mathrm{a} 1491$ & 4 & 5 & 39 & 0 & 0.19634 & 0.58904 \\
$14 \mathrm{a} 6398$ & 3 & 7 & 59 & 0 & 0.49979 & 2.64179 \\
$14 \mathrm{a} 6912$ & 3 & 8 & 67 & 0 & 0.42586 & 0.62133 \\
$14 \mathrm{a} 8662$ & 3 & 7 & 53 & 0 & 0.35779 & 0.68940 \\
$14 \mathrm{a} 13089$ & 4 & 7 & 53 & 0 & 0.15707 & 1.41371 \\
$14 \mathrm{a} 15296$ & 3 & 4 & 29 & 0 & 0.46013 & 0.58706 \\
$14 \mathrm{a} 16309$ & 3 & 7 & 53 & 0 & 0.47996 & 1.61442 \\
$14 \mathrm{a} 16437$ & 3 & 5 & 29 & 0 & 0.35419 & 1.74019 \\
$14 \mathrm{a} 18187$ & 3 & 5 & 37 & 0 & 0.38646 & 0.66073 \\
$14 \mathrm{n} 6560$ & 3 & 8 & 47 & 0 & 0.25703 & 2.88455 \\
$14 \mathrm{n} 9732$ & 3 & 7 & 53 & 0 & 0.42760 & 1.66678 \\
$14 \mathrm{n} 13886$ & 3 & 4 & 29 & 0 & 0.42839 & 2.71319 \\
$14 \mathrm{n} 14189$ & 4 & 5 & 63 & 0 & 0.39269 & 2.74889 \\
$14 \mathrm{n} 15552$ & 3 & 5 & 28 & 0 & 0.33319 & 2.80839 \\
$14 \mathrm{n} 18513$ & 3 & 8 & 83 & 0 & 0.58113 & 1.51325 \\
$14 \mathrm{n} 22071$ & 3 & 7 & 64 & 0 & 0.50884 & 2.63274 \\
$14 \mathrm{n} 22073$ & 3 & 5 & 29 & 0 & 0.50819 & 2.63339 \\
$14 \mathrm{n} 23738$ & 3 & 7 & 50 & 0 & 0.39499 & 1.69939 \\
$14 \mathrm{n} 24494$ & 3 & 8 & 61 & 0 & 0.35665 & 1.73774 \\
$14 \mathrm{n} 25903$ & 3 & 7 & 53 & 0 & 0.37524 & 1.71914 \\
\hline $15 \mathrm{a} 80928$ & 4 & 5 & 37 & 0 & 0.20569 & 0.57969 \\
$15 \mathrm{n} 77228$ & 4 & 5 & 69 & 0 & 0.42453 & 1.14625 \\
$15 \mathrm{n} 92508$ & 3 & 8 & 61 & 0 & 0.43253 & 2.70905 \\
$15 \mathrm{n} 103019$ & 5 & 6 & 103 & 0 & 0.30263 & 2.83896 \\
$15 \mathrm{n} 116110$ & 4 & 7 & 53 & 0 & 0.14398 & 2.21220 \\
\hline $16 \mathrm{a} 128851$ & 3 & 7 & 40 & 0 & 0.30079 & 2.84080 \\
$16 \mathrm{a} 151023$ & 3 & 7 & 38 & 0 & 0.26761 & 0.77958 \\
$16 \mathrm{a} 168328$ & 3 & 7 & 41 & 0 & 0.29452 & 2.84706 \\
$16 \mathrm{a} 202258$ & 3 & 7 & 40 & 0 & 0.27850 & 1.81588 \\
$16 \mathrm{a} 295212$ & 3 & 5 & 26 & 0 & 0.38847 & 1.70591 \\
$16 \mathrm{a} 312423$ & 3 & 5 & 29 & 0 & 0.38499 & 0.66219 \\
$16 \mathrm{a} 340770$ & 3 & 7 & 41 & 0 & 0.38179 & 0.66540 \\
$16 \mathrm{n} 42863$ & 4 & 5 & 39 & 0 & 0.21419 & 1.35659 \\
$16 \mathrm{n} 228473$ & 3 & 7 & 38 & 0 & 0.29088 & 1.80350 \\
$16 \mathrm{n} 390014$ & 3 & 5 & 26 & 0 & 0.35469 & 2.78689 \\
$16 \mathrm{n} 507235$ & 3 & 5 & 28 & 0 & 0.39666 & 0.65053 \\
$16 \mathrm{n} 562396$ & 3 & 5 & 28 & 0 & 0.36493 & 1.72946 \\
$16 \mathrm{n} 768985$ & 3 & 4 & 23 & 0 & 0.48481 & 1.60958 \\
$16 \mathrm{n} 982564$ & 4 & 5 & 73 & 0 & 0.44304 & 1.12775 \\
$16 \mathrm{n} 988939$ & 3 & 7 & 41 & 0 & 0.51269 & 0.53450 \\
$16 \mathrm{n} 1008347$ & 3 & 7 & 50 & 0 & 0.32150 & 0.72568 \\
\hline
\end{tabular}

Table 11: Small-crossing Lissajous knots with all frequencies greater than 2. Knot names are as in Knotscape. 


\begin{tabular}{|c|c|c|c|c|c|c|c|c|c|}
\hline knot & $p / q$ & $n_{x}$ & $n_{y}$ & $n_{z, 1}$ & $n_{z, 2}$ & $\phi_{x}$ & $\phi_{y}$ & $\phi_{z, 1}$ & $\phi_{z, 2}$ \\
\hline $3 \mathrm{a} 1$ & $3 / 1$ & 2 & 3 & 1 & 2 & 0 & $\pi / 4$ & 0.39269 & 1.66017 \\
\hline $4 \mathrm{a} 1$ & $5 / 2$ & 2 & 3 & 1 & 3 & 0 & $\pi / 4$ & 1.62773 & 5.79254 \\
\hline $5 \mathrm{a} 1$ & $7 / 2$ & 2 & 3 & 1 & 7 & 0 & $\pi / 4$ & 0.10580 & 2.49320 \\
\hline $5 \mathrm{a} 2$ & $5 / 1$ & 2 & 5 & 2 & 3 & 0 & $\pi / 4$ & 0.96046 & 4.09767 \\
\hline 6a1 & $13 / 5$ & 2 & 5 & 1 & 5 & 0 & $\pi / 4$ & 0.03573 & 2.53353 \\
\hline $6 \mathrm{a} 2$ & $11 / 3$ & 2 & 7 & 1 & 7 & 0 & $\pi / 4$ & 1.90655 & 5.01637 \\
\hline $6 \mathrm{a} 3$ & $9 / 2$ & 2 & 3 & 1 & 5 & 0 & $\pi / 4$ & 0.18165 & 1.75945 \\
\hline 7a1 & $21 / 8$ & 2 & 5 & 1 & 5 & 0 & $\pi / 4$ & 1.60021 & 5.52412 \\
\hline $7 \mathrm{a} 2$ & $19 / 7$ & 2 & 7 & 3 & 7 & 0 & $\pi / 4$ & 1.66835 & 6.11271 \\
\hline $7 \mathrm{a} 3$ & $17 / 5$ & 2 & 7 & 5 & 9 & 0 & $\pi / 4$ & 0.08774 & 5.55745 \\
\hline $7 \mathrm{a} 4$ & $11 / 2$ & 2 & 7 & 3 & 7 & 0 & $\pi / 4$ & 1.60853 & 6.27384 \\
\hline $7 \mathrm{a} 5$ & $13 / 3$ & 2 & 9 & 4 & 7 & 0 & $\pi / 4$ & 1.57817 & 4.41032 \\
\hline $7 \mathrm{a} 6$ & $15 / 4$ & 2 & 3 & 1 & 7 & 0 & $\pi / 4$ & 1.87792 & 4.64352 \\
\hline $7 \mathrm{a} 7$ & $7 / 1$ & 2 & 7 & 2 & 5 & 0 & $\pi / 4$ & 0.93991 & 0.93104 \\
\hline $8 \mathrm{a} 1$ & $31 / 12$ & 2 & 11 & 1 & 5 & 0 & $\pi / 4$ & 0.37204 & 1.78795 \\
\hline $8 a_{4}$ & $25 / 9$ & 2 & 7 & 1 & 5 & 0 & $\pi / 4$ & 2.04720 & 5.29197 \\
\hline $8 \mathrm{a} 5$ & $29 / 12$ & 2 & 5 & 3 & 5 & 0 & $\pi / 4$ & 1.59453 & 2.05821 \\
\hline $8 \mathrm{a} 6$ & $23 / 5$ & 2 & 9 & 1 & 9 & 0 & $\pi / 4$ & 0.35397 & 2.65710 \\
\hline $8 \mathrm{a} 7$ & $29 / 8$ & 2 & 9 & 1 & 5 & 0 & $\pi / 4$ & 1.47451 & 2.10447 \\
\hline $8 \mathrm{a} 8$ & $17 / 3$ & 2 & 11 & 3 & 10 & 0 & $\pi / 4$ & 0.39241 & 5.09182 \\
\hline $8 \mathrm{a} 9$ & $27 / 8$ & 2 & 9 & 1 & 5 & 0 & $\pi / 4$ & 2.03830 & 2.05668 \\
\hline $8 a 10$ & $23 / 7$ & 2 & 7 & 1 & 9 & 0 & $\pi / 4$ & 0.48400 & 5.18915 \\
\hline 8a11 & $13 / 2$ & 2 & 5 & 3 & 5 & 0 & $\pi / 4$ & 1.58524 & 0.24531 \\
\hline $8 \mathrm{a} 16$ & $25 / 7$ & 2 & 7 & 3 & 7 & 0 & $\pi / 4$ & 0.04412 & 2.25248 \\
\hline 8a17 & $19 / 4$ & 2 & 9 & 3 & 7 & 0 & $\pi / 4$ & 1.92077 & 6.06457 \\
\hline $8 a 18$ & $17 / 4$ & 2 & 7 & 1 & 5 & 0 & $\pi / 4$ & 1.42912 & 1.98797 \\
\hline $9 a 3$ & $41 / 16$ & 2 & 7 & 3 & 5 & 0 & $\pi / 4$ & 1.88608 & 4.98854 \\
\hline $9 a 8$ & $31 / 11$ & 2 & 5 & 7 & 9 & 0 & $\pi / 4$ & 0.09293 & 5.50516 \\
\hline $9 a 10$ & $39 / 16$ & 2 & 5 & 1 & 7 & 0 & $\pi / 4$ & 2.08636 & 5.17367 \\
\hline 9a12 & $49 / 18$ & 2 & 17 & 1 & 9 & 0 & $\pi / 4$ & 0.60289 & 4.56332 \\
\hline 9a13 & $55 / 21$ & 2 & 7 & 1 & 7 & 0 & $\pi / 4$ & 0.00613 & 5.43134 \\
\hline 9a14 & $39 / 14$ & 2 & 9 & 1 & 9 & 0 & $\pi / 4$ & 2.13083 & 5.89035 \\
\hline 9a15 & $47 / 13$ & 2 & 9 & 1 & 9 & 0 & $\pi / 4$ & 1.74912 & 1.92322 \\
\hline 9a16 & $45 / 19$ & 2 & 9 & 1 & 5 & 0 & $\pi / 4$ & 1.93719 & 4.94328 \\
\hline 9a17 & $37 / 8$ & 2 & 5 & 2 & 9 & 0 & $\pi / 4$ & 0.19367 & 2.96295 \\
\hline 9a19 & $41 / 11$ & 2 & 15 & 3 & 10 & 0 & $\pi / 4$ & 2.62089 & 0.60844 \\
\hline $9 \mathrm{a} 20$ & $33 / 7$ & 2 & 17 & 5 & 7 & 0 & $\pi / 4$ & 0.10752 & 5.13086 \\
\hline $9 \mathrm{a} 21$ & $43 / 12$ & 2 & 5 & 3 & 9 & 0 & $\pi / 4$ & 2.14045 & 5.61205 \\
\hline $9 \mathrm{a} 22$ & $35 / 8$ & 2 & 5 & 7 & 9 & 0 & $\pi / 4$ & 1.67486 & 1.65979 \\
\hline $9 \mathrm{a} 23$ & $27 / 5$ & 2 & 13 & 4 & 5 & 0 & $\pi / 4$ & 1.36285 & 5.38881 \\
\hline $9 a 24$ & $41 / 12$ & 2 & 11 & 1 & 7 & 0 & $\pi / 4$ & 0.44828 & 2.24339 \\
\hline $9 \mathrm{a} 26$ & $29 / 9$ & 2 & 11 & 8 & 9 & 0 & $\pi / 4$ & 1.42268 & 3.50098 \\
\hline 9a27 & $15 / 2$ & 2 & 5 & 1 & 7 & 0 & $\pi / 4$ & 0.00135 & 6.21184 \\
\hline $9 a 33$ & $31 / 7$ & 2 & 11 & 4 & 9 & 0 & $\pi / 4$ & 0.93417 & 3.86038 \\
\hline 9a34 & $37 / 10$ & 2 & 9 & 3 & 5 & 0 & $\pi / 4$ & 1.98367 & 5.56618 \\
\hline 9a35 & $21 / 4$ & 2 & 11 & 9 & 10 & 0 & $\pi / 4$ & 0.35932 & 5.18305 \\
\hline $9 a 36$ & $23 / 4$ & 2 & 9 & 3 & 5 & 0 & $\pi / 4$ & 1.65102 & 3.04593 \\
\hline 9a38 & $19 / 3$ & 2 & 13 & 1 & 7 & 0 & $\pi / 4$ & 1.93386 & 2.02910 \\
\hline $9 a 39$ & $33 / 10$ & 2 & 11 & 3 & 7 & 0 & $\pi / 4$ & 2.16159 & 2.03213 \\
\hline 9a41 & $9 / 1$ & 2 & 9 & 2 & 7 & 0 & $\pi / 4$ & 0.86114 & 0.74621 \\
\hline
\end{tabular}

Table 12: Fourier- $(1,1,2)$ descriptions of all 2-bridge knots to 9 crossings. All amplitudes are 1. Boldface entries are known to be Lissajous while italic entries might be Lissajous. All others cannot be Lissajous. Knot names are as in Knotscape. 


\begin{tabular}{|c|c|c|c|c|c|c|c|c|c|}
\hline knot & $p / q$ & $n_{x}$ & $n_{y}$ & $n_{z, 1}$ & $n_{z, 2}$ & $\phi_{x}$ & $\phi_{y}$ & $\phi_{z, 1}$ & $\phi_{z, 2}$ \\
\hline $10 \mathrm{a} 5$ & $51 / 20$ & 2 & 11 & 3 & 7 & 0 & $\pi / 4$ & 0.18595 & 2.82470 \\
\hline 10a19 & $37 / 13$ & 2 & 13 & 3 & 7 & 0 & $\pi / 4$ & 1.85642 & 1.83386 \\
\hline $10 \mathrm{a} 23$ & $49 / 20$ & 2 & 5 & 1 & 7 & 0 & $\pi / 4$ & 0.36657 & 2.47281 \\
\hline $10 \mathrm{a} 25$ & $89 / 34$ & 2 & 7 & 1 & 7 & 0 & $\pi / 4$ & 1.62228 & 5.59775 \\
\hline $10 \mathrm{a} 26$ & $61 / 22$ & 2 & 17 & 3 & 8 & 0 & $\pi / 4$ & 0.19982 & 4.43387 \\
\hline $10 \mathrm{a} 29$ & $59 / 25$ & 2 & 9 & 1 & 5 & 0 & $\pi / 4$ & 1.82942 & 5.29828 \\
\hline 10a30 & $75 / 29$ & 2 & 15 & 5 & 7 & 0 & $\pi / 4$ & 1.66311 & 4.79250 \\
\hline 10a31 & $81 / 31$ & 2 & 17 & 3 & 7 & 0 & $\pi / 4$ & 1.36514 & 5.07457 \\
\hline 10a32 & $79 / 29$ & 2 & 17 & 3 & 7 & 0 & $\pi / 4$ & 2.51922 & 6.10032 \\
\hline $10 a 33$ & $57 / 13$ & 2 & 17 & 1 & 9 & 0 & $\pi / 4$ & 1.22778 & 5.48698 \\
\hline 10a34 & $67 / 18$ & 2 & 13 & 1 & 5 & 0 & $\pi / 4$ & 1.94658 & 5.20996 \\
\hline 10a35 & $71 / 26$ & 2 & 17 & 5 & 7 & 0 & $\pi / 4$ & 2.17223 & 4.46442 \\
\hline $10 a 43$ & $47 / 11$ & 2 & 17 & 5 & 8 & 0 & $\pi / 4$ & 2.17425 & 5.50211 \\
\hline $10 \mathrm{a} 44$ & $53 / 14$ & 2 & 9 & 3 & 5 & 0 & $\pi / 4$ & 2.12967 & 5.39832 \\
\hline $10 \mathrm{a} 49$ & $53 / 23$ & 2 & 13 & 1 & 7 & 0 & $\pi / 4$ & 1.00888 & 3.57446 \\
\hline $10 \mathrm{a} 52$ & $73 / 27$ & 2 & 17 & 3 & 7 & 0 & $\pi / 4$ & 2.90021 & 2.80121 \\
\hline $10 \mathrm{a} 53$ & $63 / 17$ & 2 & 17 & 1 & 9 & 0 & $\pi / 4$ & 0.46162 & 2.91082 \\
\hline $10 \mathrm{a} 54$ & $53 / 12$ & 2 & 5 & 5 & 7 & 0 & $\pi / 4$ & 0.00438 & 1.96518 \\
\hline $10 \mathrm{a} 55$ & $69 / 19$ & 2 & 13 & 3 & 7 & 0 & $\pi / 4$ & 2.09996 & 5.23165 \\
\hline $10 \mathrm{a} 56$ & $33 / 5$ & 2 & 19 & 5 & 6 & 0 & $\pi / 4$ & 0.37923 & 4.72239 \\
\hline $10 \mathrm{a} 57$ & $59 / 18$ & 2 & 21 & 3 & 8 & 0 & $\pi / 4$ & 0.38316 & 4.48117 \\
\hline $10 a 58$ & $71 / 21$ & 2 & 13 & 6 & 9 & 0 & $\pi / 4$ & 1.04484 & 0.64343 \\
\hline $10 \mathrm{a} 59$ & $23 / 3$ & 2 & 23 & 2 & 7 & 0 & $\pi / 4$ & 0.57078 & 0.10280 \\
\hline $10 \mathrm{a} 60$ & $45 / 14$ & 2 & 17 & 1 & 9 & 0 & $\pi / 4$ & 1.72208 & 1.81144 \\
\hline $10 a 61$ & $65 / 19$ & 2 & 17 & 2 & 5 & 0 & $\pi / 4$ & 1.05935 & 4.53128 \\
\hline 10a63 & $55 / 12$ & 2 & 11 & 1 & 7 & 0 & $\pi / 4$ & 2.07884 & 1.80942 \\
\hline $10 \mathrm{a} 64$ & $45 / 8$ & 2 & 17 & 5 & 9 & 0 & $\pi / 4$ & 1.41312 & 1.49892 \\
\hline $10 \mathrm{a} 65$ & $43 / 8$ & 2 & 19 & 1 & 7 & 0 & $\pi / 4$ & 1.64470 & 4.03256 \\
\hline 10a68 & $43 / 9$ & 2 & 13 & 9 & 10 & 0 & $\pi / 4$ & 0.30693 & 1.31007 \\
\hline $10 \mathrm{a} 69$ & $57 / 16$ & 2 & 9 & 3 & 5 & 0 & $\pi / 4$ & 0.02223 & 2.46173 \\
\hline 10a70 & $37 / 7$ & 2 & 19 & 5 & 7 & 0 & $\pi / 4$ & 0.36129 & 4.69287 \\
\hline $10 a^{r} 71$ & $55 / 16$ & 2 & 13 & 3 & 5 & 0 & $\pi / 4$ & 1.15846 & 5.71558 \\
\hline $10 \mathrm{a} 74$ & $35 / 11$ & 2 & 13 & 5 & 10 & 0 & $\pi / 4$ & 0.31052 & 4.18157 \\
\hline $10 \mathrm{a} 75$ & $17 / 2$ & 2 & 5 & 2 & 7 & 0 & $\pi / 4$ & 0.00295 & 3.10669 \\
\hline 10a107 & $41 / 9$ & 2 & 9 & 5 & 9 & 0 & $\pi / 4$ & 0.11322 & 5.44496 \\
\hline 10a108 & $51 / 11$ & 2 & 17 & 2 & 7 & 0 & $\pi / 4$ & 1.01343 & 4.49878 \\
\hline 10a109 & $65 / 18$ & 2 & 11 & 2 & 5 & 0 & $\pi / 4$ & 0.22667 & 5.30925 \\
\hline 10a110 & $39 / 7$ & 2 & 19 & 4 & 5 & 0 & $\pi / 4$ & 1.10277 & 4.42691 \\
\hline 10a111 & $61 / 17$ & 2 & 19 & 4 & 5 & 0 & $\pi / 4$ & 1.35067 & 1.23088 \\
\hline 10a112 & $49 / 13$ & 2 & 17 & 5 & 7 & 0 & $\pi / 4$ & 0.06896 & 1.24086 \\
\hline 10a113 & $27 / 4$ & 2 & 17 & 4 & 7 & 0 & $\pi / 4$ & 1.05187 & 2.20380 \\
\hline 10a114 & $29 / 5$ & 2 & 19 & 5 & 7 & 0 & $\pi / 4$ & 0.18690 & 4.45167 \\
\hline $10 a 115$ & $47 / 10$ & 2 & 11 & 7 & 10 & 0 & $\pi / 4$ & 0.50632 & 4.53216 \\
\hline 10a116 & $43 / 10$ & 2 & 15 & 7 & 9 & 0 & $\pi / 4$ & 0.09320 & 1.94013 \\
\hline $10 a 117$ & $25 / 4$ & 2 & 7 & 1 & 5 & 0 & $\pi / 4$ & 2.15907 & 2.20357 \\
\hline
\end{tabular}

Table 13: Fourier-(1,1,2) descriptions of all 2-bridge knots with 10 crossings. All amplitudes are 1. Boldface entries are known to be Lissajous while italic entries might be Lissajous. All others cannot be Lissajous. Knot names are as in Knotscape. 


\begin{tabular}{|l|cccccccc|}
\hline knot & $n_{x}$ & $n_{y}$ & $n_{z, 1}$ & $n_{z, 2}$ & $\phi_{x}$ & $\phi_{y}$ & $\phi_{z, 1}$ & $\phi_{z, 2}$ \\
\hline 8a2 & 3 & 4 & 1 & 7 & 0 & $\pi / 6$ & 1.63362 & 2.03575 \\
8a3 & 3 & 5 & 6 & 13 & 0 & $\pi / 6$ & 0.56548 & 2.03575 \\
8a12 & 3 & 4 & 3 & 5 & 0 & $\pi / 6$ & 1.04300 & 0.80424 \\
8a13 & 3 & 4 & 1 & 9 & 0 & $\pi / 6$ & 0.26389 & 1.58336 \\
8a14 & 3 & 4 & 7 & 14 & 0 & $\pi / 6$ & 1.28176 & 1.78442 \\
8a15 & 3 & 7 & 1 & 10 & 0 & $\pi / 6$ & 1.64619 & 2.31221 \\
8n1 & 3 & 4 & 1 & 14 & 0 & $\pi / 6$ & 1.94778 & 2.76460 \\
8n2 & 3 & 4 & 1 & 5 & 0 & $\pi / 6$ & 0.05026 & 2.23681 \\
8n3 & 3 & 4 & 1 & 3 & 0 & $\pi / 6$ & 0.26389 & 1.58336 \\
\hline 9a1 & 3 & 5 & 7 & 10 & 0 & $\pi / 6$ & 2.29964 & 0.03769 \\
9a2 & 3 & 7 & 1 & 6 & 0 & $\pi / 6$ & 0.35185 & 1.05557 \\
9a4 & 3 & 4 & 1 & 14 & 0 & $\pi / 6$ & 1.33203 & 2.27451 \\
9a5 & 3 & 8 & 1 & 8 & 0 & $\pi / 6$ & 0.65345 & 1.70902 \\
9a6 & 3 & 7 & 1 & 10 & 0 & $\pi / 6$ & 1.88495 & 2.43787 \\
9a7 & 3 & 4 & 1 & 14 & 0 & $\pi / 6$ & 2.03575 & 2.37504 \\
9a9 & 3 & 7 & 4 & 15 & 0 & $\pi / 6$ & 1.15610 & 0.76654 \\
9a11 & 3 & 5 & 9 & 14 & 0 & $\pi / 6$ & 1.04300 & 1.33203 \\
9a18 & 3 & 4 & 2 & 7 & 0 & $\pi / 6$ & 2.37504 & 2.03575 \\
9a25 & 3 & 7 & 2 & 14 & 0 & $\pi / 6$ & 0.98017 & 0.37699 \\
9a28 & 3 & 7 & 4 & 5 & 0 & $\pi / 6$ & 1.28176 & 0.45238 \\
9a29 & 4 & 7 & 2 & 13 & 0 & $\pi / 8$ & 0.26389 & 2.07345 \\
9a30 & 3 & 7 & 8 & 9 & 0 & $\pi / 6$ & 0.23876 & 0.77911 \\
9a31 & 3 & 4 & 10 & 11 & 0 & $\pi / 6$ & 1.38230 & 1.87238 \\
9a32 & 3 & 7 & 4 & 13 & 0 & $\pi / 6$ & 0.46495 & 1.20637 \\
9a37 & 3 & 4 & 2 & 11 & 0 & $\pi / 6$ & 1.04300 & 2.62637 \\
9a40 & 3 & 7 & 4 & 13 & 0 & $\pi / 6$ & 1.06814 & 1.06814 \\
9n1 & 3 & 4 & 1 & 14 & 0 & $\pi / 6$ & 0.05026 & 0.27646 \\
9n2 & 3 & 5 & 4 & 7 & 0 & $\pi / 6$ & 0.15079 & 1.99805 \\
9n3 & 3 & 8 & 1 & 6 & 0 & $\pi / 6$ & 0.76654 & 0.95504 \\
9n4 & 3 & 4 & 2 & 11 & 0 & $\pi / 6$ & 0.35185 & 2.51327 \\
9n5 & 3 & 4 & 1 & 4 & 0 & $\pi / 6$ & 1.33203 & 2.09858 \\
9n6 & 3 & 4 & 2 & 13 & 0 & $\pi / 6$ & 0.08796 & 2.48814 \\
9n7 & 3 & 7 & 2 & 9 & 0 & $\pi / 6$ & 2.62637 & 1.05557 \\
9n8 & 3 & 7 & 4 & 13 & 0 & $\pi / 6$ & 0.05026 & 0.18849 \\
\hline
\end{tabular}

Table 14: Fourier-(1,1,2) descriptions of non 2-bridge knots up to 9 crossings. All amplitudes are 1. Knot names are as in Knotscape. 


\begin{tabular}{|l|cccccccc|}
\hline knot & $n_{x}$ & $n_{y}$ & $n_{z, 1}$ & $n_{z, 2}$ & $\phi_{x}$ & $\phi_{y}$ & $\phi_{z, 1}$ & $\phi_{z, 2}$ \\
\hline 10a1 & 3 & 4 & 5 & 9 & 0 & $\pi / 6$ & 1.04300 & 1.58336 \\
10a2 & 3 & 8 & 4 & 13 & 0 & $\pi / 6$ & 0.70371 & 0.08796 \\
10a3 & 3 & 8 & 7 & 14 & 0 & $\pi / 6$ & 2.70176 & 0.23876 \\
10a4 & 3 & 5 & 4 & 13 & 0 & $\pi / 6$ & 2.34991 & 2.34991 \\
10a7 & 3 & 5 & 8 & 11 & 0 & $\pi / 6$ & 0.01256 & 1.60849 \\
10a9 & 3 & 8 & 5 & 6 & 0 & $\pi / 6$ & 0.27646 & 1.58336 \\
10a10 & 3 & 10 & 2 & 11 & 0 & $\pi / 6$ & 2.48814 & 2.94053 \\
10a11 & 3 & 8 & 3 & 14 & 0 & $\pi / 6$ & 0.05026 & 1.01787 \\
10a12 & 3 & 8 & 2 & 5 & 0 & $\pi / 6$ & 1.40743 & 1.67132 \\
10a14 & 3 & 8 & 2 & 7 & 0 & $\pi / 6$ & 0.33929 & 2.81486 \\
10a15 & 4 & 7 & 1 & 12 & 0 & $\pi / 8$ & 1.01787 & 0.67858 \\
10a16 & 4 & 7 & 1 & 12 & 0 & $\pi / 8$ & 0.20106 & 0.66601 \\
10a17 & 3 & 8 & 5 & 15 & 0 & $\pi / 6$ & 0.22619 & 1.06814 \\
10a18 & 3 & 8 & 4 & 9 & 0 & $\pi / 6$ & 0.17592 & 1.58336 \\
10a20 & 3 & 4 & 1 & 7 & 0 & $\pi / 6$ & 0.22619 & 1.47026 \\
10a21 & 3 & 8 & 1 & 10 & 0 & $\pi / 6$ & 0.82938 & 2.71433 \\
10a22 & 3 & 8 & 1 & 10 & 0 & $\pi / 6$ & 2.46300 & 2.75203 \\
10a28 & 4 & 7 & 1 & 14 & 0 & $\pi / 8$ & 0.43982 & 0.11309 \\
10a36 & 4 & 5 & 1 & 14 & 0 & $\pi / 8$ & 0.82938 & 1.60849 \\
10a37 & 3 & 5 & 7 & 14 & 0 & $\pi / 6$ & 0.07539 & 2.90283 \\
10a38 & 3 & 7 & 2 & 11 & 0 & $\pi / 6$ & 0.66601 & 0.01256 \\
10a42 & 3 & 10 & 5 & 8 & 0 & $\pi / 6$ & 2.61380 & 0.42725 \\
10a47 & 3 & 4 & 5 & 14 & 0 & $\pi / 6$ & 2.19911 & 1.96035 \\
10a48 & 3 & 8 & 5 & 10 & 0 & $\pi / 6$ & 2.02318 & 1.39486 \\
10a50 & 3 & 7 & 1 & 8 & 0 & $\pi / 6$ & 1.60849 & 1.09327 \\
10a51 & 3 & 7 & 2 & 4 & 0 & $\pi / 6$ & 2.56353 & 5.45380 \\
10a62 & 5 & 6 & 4 & 11 & 0 & $\pi / 10$ & 0.27646 & 0.59061 \\
10a66 & 3 & 5 & 2 & 11 & 0 & $\pi / 6$ & 1.52053 & 1.85982 \\
10a67 & 3 & 8 & 2 & 13 & 0 & $\pi / 6$ & 1.40743 & 3.12902 \\
\hline
\end{tabular}

Table 15: Fourier- $(1,1,2)$ descriptions of alternating non 2-bridge knots with 10 crossings. All amplitudes are 1. Knot names are as in Knotscape. 


\begin{tabular}{|l|cccccccc|}
\hline knot & $n_{x}$ & $n_{y}$ & $n_{z, 1}$ & $n_{z, 2}$ & $\phi_{x}$ & $\phi_{y}$ & $\phi_{z, 1}$ & $\phi_{z, 2}$ \\
\hline 10a72 & 4 & 5 & 7 & 14 & 0 & $\pi / 8$ & 2.47557 & 1.73415 \\
10a93 & 3 & 5 & 2 & 10 & 0 & $\pi / 6$ & 1.98548 & 0.16336 \\
10a76 & 3 & 7 & 6 & 11 & 0 & $\pi / 6$ & 1.58336 & 1.05557 \\
10aa77 & 5 & 6 & 3 & 14 & 0 & $\pi / 10$ & 0.30159 & 1.75929 \\
10a78 & 5 & 6 & 1 & 12 & 0 & $\pi / 10$ & 1.45769 & 1.26920 \\
10a80 & 3 & 10 & 4 & 15 & 0 & $\pi / 6$ & 1.97292 & 0.31415 \\
10a82 & 5 & 6 & 4 & 11 & 0 & $\pi / 10$ & 0.37699 & 2.86513 \\
10a84 & 4 & 7 & 6 & 13 & 0 & $\pi / 8$ & 0.31415 & 1.73415 \\
10a85 & 3 & 4 & 7 & 14 & 0 & $\pi / 6$ & 1.08070 & 2.04831 \\
10a87 & 4 & 7 & 2 & 3 & 0 & $\pi / 8$ & 1.43256 & 2.14884 \\
10a99 & 3 & 4 & 3 & 13 & 0 & $\pi / 6$ & 0.18849 & 1.48283 \\
10a90 & 5 & 6 & 6 & 7 & 0 & $\pi / 10$ & 0.02513 & 1.53309 \\
10a91 & 4 & 7 & 1 & 14 & 0 & $\pi / 8$ & 0.70371 & 0.05026 \\
10a92 & 3 & 4 & 7 & 14 & 0 & $\pi / 6$ & 1.09327 & 2.46300 \\
10a93 & 3 & 8 & 2 & 13 & 0 & $\pi / 6$ & 1.57079 & 0.69115 \\
10a94 & 3 & 4 & 7 & 10 & 0 & $\pi / 6$ & 1.28176 & 1.36973 \\
10a95 & 4 & 7 & 4 & 13 & 0 & $\pi / 8$ & 1.58336 & 0.06283 \\
10a96 & 3 & 4 & 7 & 13 & 0 & $\pi / 6$ & 1.63362 & 5.17734 \\
10a97 & 3 & 8 & 2 & 7 & 0 & $\pi / 6$ & 1.35716 & 2.36247 \\
10a99 & 3 & 8 & 2 & 7 & 0 & $\pi / 6$ & 1.36973 & 2.63893 \\
10a100 & 3 & 10 & 4 & 7 & 0 & $\pi / 6$ & 1.73415 & 1.41999 \\
10a101 & 5 & 6 & 3 & 8 & 0 & $\pi / 10$ & 0.06283 & 2.57610 \\
10a102 & 3 & 4 & 1 & 13 & 0 & $\pi / 6$ & 2.02318 & 1.24407 \\
10a103 & 3 & 5 & 9 & 13 & 0 & $\pi / 6$ & 0.75398 & 2.57610 \\
10a105 & 3 & 4 & 10 & 13 & 0 & $\pi / 6$ & 1.77185 & 2.86513 \\
10a119 & 4 & 7 & 10 & 15 & 0 & $\pi / 8$ & 0.45238 & 1.25663 \\
10a121 & 3 & 5 & 3 & 7 & 0 & $\pi / 6$ & 1.04300 & 0.85451 \\
10a122 & 4 & 7 & 7 & 9 & 0 & $\pi / 8$ & 1.28176 & 3.99610 \\
10a123 & 3 & 7 & 2 & 6 & 0 & $\pi / 6$ & 0.57805 & 4.31026 \\
\hline
\end{tabular}

Table 16: Fourier- $(1,1,2)$ descriptions of alternating non 2-bridge knots with 10 crossings. All amplitudes are 1. Knot names are as in Knotscape. 


\begin{tabular}{|c|c|c|c|c|c|c|c|c|}
\hline knot & $n_{x}$ & $n_{y}$ & $n_{z, 1}$ & $n_{z, 2}$ & $\phi_{x}$ & $\phi_{y}$ & $\phi_{z, 1}$ & $\phi_{z, 2}$ \\
\hline 10n1 & 3 & 4 & 3 & 7 & 0 & $\pi / 6$ & 1.04300 & 1.47026 \\
\hline 10n2 & 3 & 4 & 5 & 14 & 0 & $\pi / 6$ & 0.99274 & 2.87769 \\
\hline 10n3 & 3 & 4 & 1 & 5 & 0 & $\pi / 6$ & 1.94778 & 5.88106 \\
\hline 10n4 & 3 & 4 & 6 & 11 & 0 & $\pi / 6$ & 0.35185 & 0.18849 \\
\hline 10n5 & 3 & 4 & 1 & 10 & 0 & $\pi / 6$ & 1.11840 & 2.37504 \\
\hline 10n6 & 5 & 6 & 1 & 4 & 0 & $\pi / 10$ & 1.60849 & 1.93522 \\
\hline 10n7 & 3 & 7 & 3 & 4 & 0 & $\pi / 6$ & 1.04300 & 0.52778 \\
\hline 10n8 & 3 & 5 & 4 & 13 & 0 & $\pi / 6$ & 0.75398 & 2.81486 \\
\hline 10n9 & 3 & 8 & 2 & 7 & 0 & $\pi / 6$ & 0.47752 & 1.45769 \\
\hline 10n10 & 3 & 8 & 2 & 5 & 0 & $\pi / 6$ & 1.99805 & 2.06088 \\
\hline 10n11 & 4 & 7 & 3 & 8 & 0 & $\pi / 8$ & 1.26920 & 0.79168 \\
\hline 10n12 & 3 & 8 & 2 & 7 & 0 & $\pi / 6$ & 1.39486 & 2.85256 \\
\hline 10n13 & 3 & 5 & 2 & 11 & 0 & $\pi / 6$ & 1.53309 & 1.28176 \\
\hline 10n14 & 3 & 5 & 2 & 9 & 0 & $\pi / 6$ & 0.33929 & 1.58336 \\
\hline 10n15 & 3 & 8 & 2 & 3 & 0 & $\pi / 6$ & 0.52778 & 1.58336 \\
\hline 10n16 & 3 & 8 & 6 & 7 & 0 & $\pi / 6$ & 0.76654 & 0.52778 \\
\hline 10n17 & 3 & 10 & 1 & 4 & 0 & $\pi / 6$ & 0.47752 & 1.70902 \\
\hline 10n18 & 3 & 4 & 1 & 10 & 0 & $\pi / 6$ & 2.02318 & 1.91008 \\
\hline 10n19 & 3 & 5 & 2 & 7 & 0 & $\pi / 6$ & 1.25663 & 2.04831 \\
\hline 10n20 & 3 & 4 & 5 & 10 & 0 & $\pi / 6$ & 0.62831 & 1.93522 \\
\hline 10n21 & 3 & 5 & 2 & 3 & 0 & $\pi / 6$ & 0.31415 & 1.58336 \\
\hline 10n22 & 3 & 10 & 4 & 13 & 0 & $\pi / 6$ & 0.02513 & 0.02513 \\
\hline $10 \mathrm{n} 23$ & 3 & 7 & 8 & 11 & 0 & $\pi / 6$ & 0.05026 & 1.28176 \\
\hline 10n24 & 3 & 4 & 7 & 14 & 0 & $\pi / 6$ & 1.74672 & 1.48283 \\
\hline $10 \mathrm{n} 25$ & 3 & 7 & 1 & 12 & 0 & $\pi / 6$ & 2.09858 & 1.58336 \\
\hline $10 \mathrm{n} 26$ & 3 & 7 & 5 & 6 & 0 & $\pi / 6$ & 0.69115 & 1.58336 \\
\hline $10 \mathrm{n} 27$ & 4 & 5 & 1 & 5 & 0 & $\pi / 8$ & 0.03769 & 4.80035 \\
\hline $10 \mathrm{n} 28$ & 3 & 4 & 7 & 11 & 0 & $\pi / 6$ & 0.05026 & 2.23681 \\
\hline $10 \mathrm{n} 29$ & 3 & 5 & 2 & 11 & 0 & $\pi / 6$ & 1.92265 & 2.09858 \\
\hline 10n30 & 3 & 7 & 2 & 4 & 0 & $\pi / 6$ & 0.38955 & 1.52053 \\
\hline 10n31 & 3 & 7 & 4 & 15 & 0 & $\pi / 6$ & 0.52778 & 0.75398 \\
\hline 10n32 & 3 & 5 & 7 & 10 & 0 & $\pi / 6$ & 0.41469 & 2.31221 \\
\hline 10n33 & 3 & 5 & 2 & 9 & 0 & $\pi / 6$ & 1.45769 & 1.58336 \\
\hline 10n34 & 3 & 8 & 1 & 12 & 0 & $\pi / 6$ & 0.66601 & 1.58336 \\
\hline 10n35 & 3 & 4 & 10 & 13 & 0 & $\pi / 6$ & 0.07539 & 3.00336 \\
\hline 10n36 & 4 & 5 & 1 & 4 & 0 & $\pi / 8$ & 0.18849 & 1.18123 \\
\hline 10n37 & 3 & 8 & 1 & 4 & 0 & $\pi / 6$ & 2.22424 & 0.22619 \\
\hline 10n38 & 3 & 4 & 5 & 10 & 0 & $\pi / 6$ & 2.55097 & 1.49539 \\
\hline 10n39 & 3 & 5 & 3 & 10 & 0 & $\pi / 6$ & 1.58336 & 0.52778 \\
\hline $10 \mathrm{n} 40$ & 3 & 5 & 7 & 14 & 0 & $\pi / 6$ & 1.00530 & 2.55097 \\
\hline 10n41 & 3 & 10 & 2 & 7 & 0 & $\pi / 6$ & 2.57610 & 2.78973 \\
\hline 10n42 & 3 & 10 & 1 & 8 & 0 & $\pi / 6$ & 1.43256 & 0.79168 \\
\hline
\end{tabular}

Table 17: Fourier-(1,1,2) descriptions of nonalternating non 2-bridge knots with 10 crossings. All amplitudes are 1. Knot names are as in Knotscape. 


\begin{tabular}{|l|l|cccccccc|}
\hline torus knot & knot & $n_{x}$ & $n_{y}$ & $n_{z, 1}$ & $n_{z, 2}$ & $\phi_{x}$ & $\phi_{y}$ & $\phi_{z, 1}$ & $\phi_{z, 2}$ \\
\hline$T_{2,3}$ & $3 \mathrm{a} 1$ & 2 & 3 & 1 & 2 & 0 & $\pi / 4$ & $\pi / 2$ & $\pi / 4$ \\
$T_{2,5}$ & $5 \mathrm{a} 2$ & 2 & 5 & 2 & 3 & 0 & $\pi / 4$ & $\pi / 2$ & $\pi / 4$ \\
$T_{2,7}$ & $7 \mathrm{a} 7$ & 2 & 7 & 2 & 5 & 0 & $\pi / 4$ & $\pi / 2$ & $\pi / 4$ \\
$T_{2,9}$ & $9 \mathrm{a} 41$ & 2 & 9 & 2 & 7 & 0 & $\pi / 4$ & $\pi / 2$ & $\pi / 4$ \\
$T_{2,11}$ & $11 \mathrm{a} 367$ & 2 & 11 & 2 & 9 & 0 & $\pi / 4$ & $\pi / 2$ & $\pi / 4$ \\
$T_{2,13}$ & $13 \mathrm{a} 4878$ & 2 & 13 & 2 & 11 & 0 & $\pi / 4$ & $\pi / 2$ & $\pi / 4$ \\
$T_{2,15}$ & $15 \mathrm{a} 85263$ & 2 & 15 & 2 & 13 & 0 & $\pi / 4$ & $\pi / 2$ & $\pi / 4$ \\
$T_{3,4}$ & $8 \mathrm{n} 3$ & 3 & 4 & 1 & 3 & 0 & $\pi / 6$ & 0.26389 & 1.58336 \\
$T_{3,5}$ & $10 \mathrm{n} 21$ & 3 & 5 & 2 & 3 & 0 & $\pi / 6$ & 0.31415 & 1.58336 \\
$T_{3,7}$ & $14 \mathrm{n} 21881$ & 3 & 7 & 3 & 4 & 0 & $\pi / 6$ & 1.57079 & 0.37699 \\
$T_{4,5}$ & $15 \mathrm{n} 41185$ & 4 & 5 & 1 & 4 & 0 & $\pi / 8$ & 0.40212 & 1.58336 \\
$T_{3,8}$ & $16 \mathrm{n} 783154$ & 3 & 8 & 3 & 5 & 0 & $\pi / 6$ & 1.57079 & 0.40212 \\
\hline
\end{tabular}

Table 18: Fourier- $(1,1,2)$ descriptions of all torus knots up to 16 crossings. All amplitudes are 1 . Knot names are as in Knotscape. 